\title{
Metabolic profile of women with PCOS in Brazil: a systematic review and meta-analysis
}

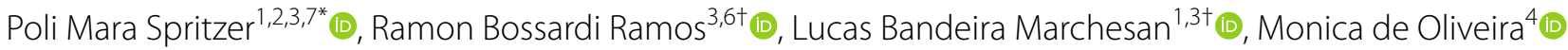
and Enrico Carmina ${ }^{5}$ (D)

\begin{abstract}
Background: Polycystic ovary syndrome (PCOS) is a common endocrine disease affecting women of reproductive age and associated with reproductive and metabolic dysfunction. Few studies are available regarding metabolic traits in Brazilian women with PCOS. The aim of this systematic review and meta-analysis was to summarize the available evidence regarding metabolic traits and comorbidities in Brazilian women with polycystic ovary syndrome (PCOS).

Methods: We systematically searched PubMed, Cochrane Central Register of Controlled Trials, and Embase for crosssectional, case-control, or cohort studies focusing on populations of different regions from Brazil, published until July 31, 2019. Studies were selected if they reported PCOS diagnostic criteria. Studies without a control group were included if they presented relevant metabolic data.

Results: Of 4856 studies initially identified, 27 were included in the systematic review and 12 were included in the meta-analysis, for a total of 995 women with PCOS defined by Rotterdam criteria and 2275 controls from different regions of Brazil. Obesity, metabolic syndrome and IGT were prevalent, and standard mean differences for BMI (SMD $0.67,95 \% \mathrm{Cl}, 0.29,1.05$ ), waist circumference (SMD 0.22, 95\% Cl 0.02, 0.41), systolic (SMD 0.66, 95\% Cl 0.30, 1.01) and diastolic blood pressure (SMD $0.55,95 \% \mathrm{Cl} 0.24,0.87$ ), glucose (SMD 0.21, 95\% Cl 0.04, 0.38) and HOMA (SMD 0.78, 95\% $\mathrm{Cl} 0.52,1.04)$ were significantly higher in Brazilian women with PCOS compared to controls. Lipid profile was more adverse in PCOS vs. non-PCOS women. Between-study heterogeneities were low/moderate for glucose and HOMA and moderate/high for the other variables.

Conclusions: The data of this systematic review and meta-analysis indicate that Brazilian women with PCOS have a worse metabolic profile than women without PCOS with no important regional differences. The prevalence of metabolic changes is intermediate in Brazil vs. other countries.
\end{abstract}

Keywords: Polycystic ovary syndrome, Obesity, Blood pressure, Insulin resistance, Metabolic abnormalities, Brazil

\section{Background}

Polycystic ovary syndrome (PCOS) is the most common endocrine disorder among women of reproductive age $[1$, 2]. Despite an uncertain etiology, there is strong evidence

\footnotetext{
*Correspondence: spritzer@ufrgs.br

${ }^{\dagger}$ Ramon Bossardi Ramos and Lucas Bandeira Marchesan contributed equally to this work

${ }^{7}$ Division of Endocrinology, Hospital de Clínicas de Porto Alegre, Rua Ramiro Barcelos, 2350, Porto Alegre, RS 90035-003, Brazil

Full list of author information is available at the end of the article
}

that complex interactions between genetic, environmental, and behavioral factors contribute to the onset and to the heterogeneous expression of the syndrome [3]. In fact, not only does the syndrome involve several different phenotypes; the prevalence of the different phenotypes also varies according to ethnic groups [4-11].

Evidence from various geographic regions indicates that the metabolic features of PCOS are particularly influenced by ethnic background and behavioral characteristics. For example, obesity is particularly common in women with PCOS from the U.S $[8,12-15]$, but

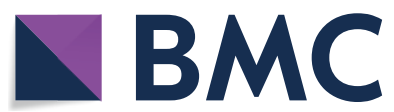

(c) The Author(s) 2021. This article is licensed under a Creative Commons Attribution 4.0 International License, which permits use, sharing, adaptation, distribution and reproduction in any medium or format, as long as you give appropriate credit to the original author(s) and the source, provide a link to the Creative Commons licence, and indicate if changes were made. The images or other third party material in this article are included in the article's Creative Commons licence, unless indicated otherwise in a credit line to the material. If material is not included in the article's Creative Commons licence and your intended use is not permitted by statutory regulation or exceeds the permitted use, you will need to obtain permission directly from the copyright holder. To view a copy of this licence, visit http://creativeco mmons.org/licenses/by/4.0/. The Creative Commons Public Domain Dedication waiver (http://creativecommons.org/publicdomain/ zero/1.0/) applies to the data made available in this article, unless otherwise stated in a credit line to the data. 
relatively uncommon in Eastern Asia [16] and in Mediterranean countries [5-7]. Impaired fasting glucose, impaired glucose tolerance, and type 2 diabetes seem particularly common in India $[17,18]$ and in the U.S. $[13,14]$ and relatively uncommon in Mediterranean countries [6,7]. A similar pattern has been reported for the prevalence of metabolic syndrome and dyslipidemia $[7,14,19]$.

In contrast, only limited information is available regarding the phenotype distribution and the metabolic expression of PCOS in some other regions, such as Brazil, a large country representing a range of ethnic backgrounds. Brazil also presents socio-economic disparities among its five regions. Total Brazilian population, according to 2020 national data, is of $211,755,692$ inhabitants, with $83 \%$ distributed in the Northeast, Southeast and South (https://www.ibge.gov.br/estat isticas/sociais.html). Regarding education, inhabitants of the southeast region have the better access to education, with $28.9 \%$ of population with 25 years old or more having concluded school, while the lowest index is in the northeast region, 23.5\%. Regarding economic status, the median monthly income per person in the South is U\$211.3, U\$187.4 in Southeast, U\$184.8 in Midwest, U\$94.6 in North and U\$ 93.7 in Northeast. However, there is great variation in incomes according to social classes, in the whole territory.

Therefore, the aim of the present systematic review and meta-analysis was to examine the available evidence regarding the prevalence of metabolic alterations in Brazilian women with PCOS.

\section{Methods}

\section{Search strategy and study selection}

This systematic review and meta-analysis was registered in PROSPERO under number CRD42016038537. PubMed, Cochrane Central Register of Controlled Trials, and Embase were searched for cross-sectional, case-control, cohort, and prevalence studies published until July, 2019 and including populations from different regions of Brazil. No limits were set on publication date or language. Medical subject headings (MeSH) used in the search are presented as Additional file 1. Additional searches were performed in recent review articles and original studies with a focus on PCOS.

Studies were selected for the present review if they provided a clear definition of the criteria used for diagnosis of PCOS and analyzed of at least one of the following variables: body mass index (BMI), waist circumference (WC), blood pressure, lipid profile, glucose, HOMA-IR, metabolic syndrome (MetS), diabetes mellitus (DM), prevalence of PCOS, and milder phenotypes.

\section{Data extraction and quality control assessment}

Two reviewers (RBR and PMS) independently screened titles/abstracts for selection of articles for full-text review. Disagreements were resolved by consensus discussion. The full text of selected articles was independently reviewed by the two authors. If selected articles were published in other languages than English or Portuguese a translate site would be used. If data were duplicated or reported more than once, the most complete study was chosen. If the required data were not located in the published article, authors were contacted to provide the missing information.

The following information was extracted from studies: name of authors, publication year, country, type of study, population characteristics, diagnostic criteria, total population, and outcomes of interest in PCOS and control group. Three authors extracted the data from each report independently. The Newcastle-Ottawa scale (NOS) was used to assess the quality of the observational studies included in the meta-analyses.

\section{Statistical analysis}

The standardized mean difference (SMD) with 95\% confidence interval $(\mathrm{CI})$ was estimated using a DerSimonian and Laird (DL) random effects model. A p value of less than 0.05 was considered as statistically significant. Variables of interest were included in the meta-analysis if they were present in at least two studies.

We assessed heterogeneity from the Mantel-Haenszel model and $\mathrm{I}^{2}$ values (the percentage of variance in the pooled estimate due to between-study differences), with $\mathrm{I}^{2}>50 \%$ suggesting moderate heterogeneity and $\mathrm{p}<0.10$ in Cochran's $\mathrm{Q}$ test indicating significant heterogeneity[20]. The risk of publication bias was assessed using funnel plot graphics, analyzed both visually and with the Egger test. The significance of the intercept was evaluated by the t test, with $\mathrm{p}<0.10$ indicating significant publication bias [21].

Statistical analyses were performed using $\mathrm{R}$ version 3.4.3. (2017-11-30) (http://www.r-project.org). The metafor package for doing meta-analysis was used within the R environment. Graphs were also created using metafor[22].

\section{Results \\ Flowchart of study selection}

Figure 1 provides details of the study selection. Our search yielded 4856 articles, of which 27 studies, all observational, were eligible for inclusion in the systematic review, 14 cross-sectional, 12 case-control studies and 01 cohort (Table 1). All articles were published 


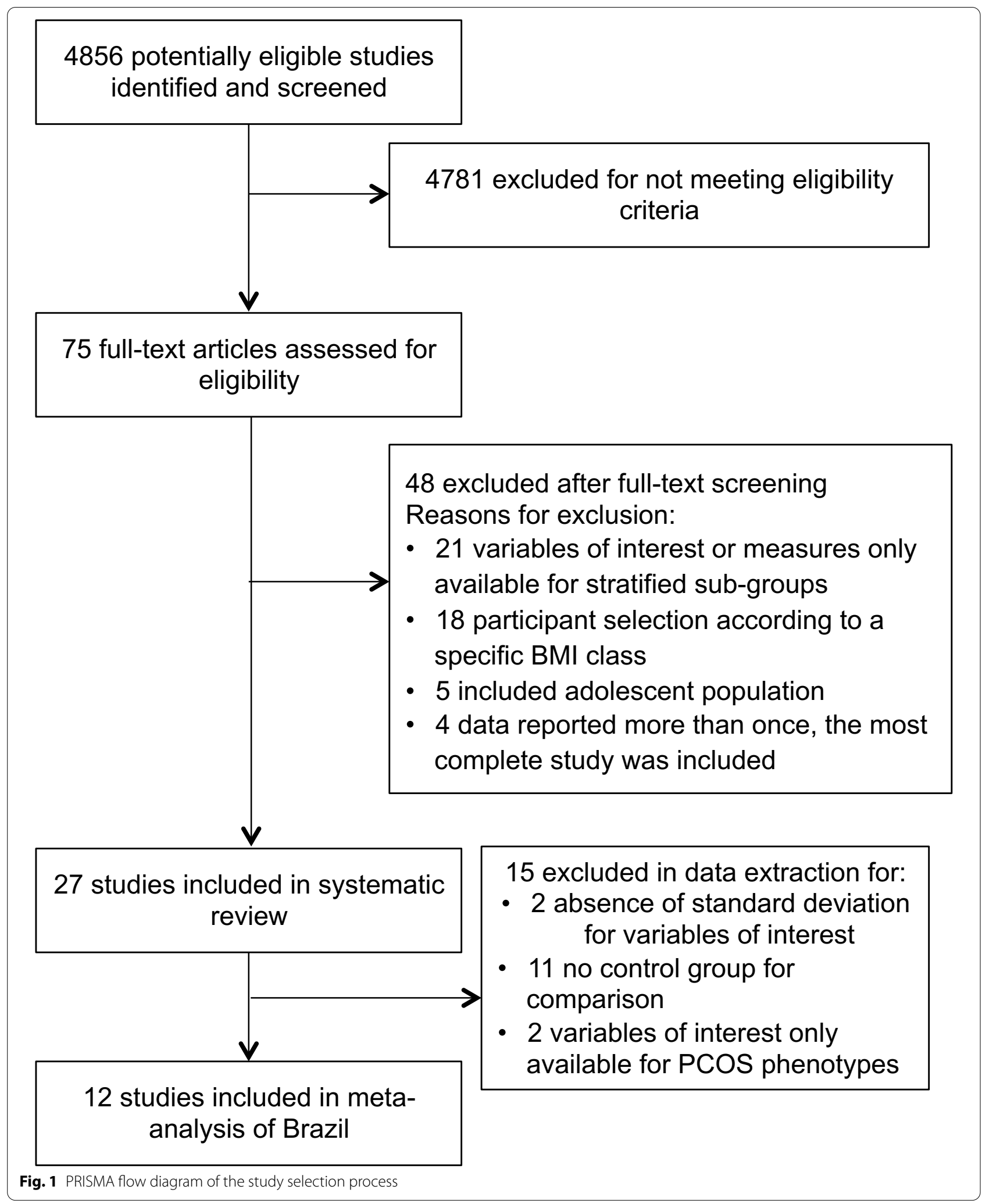


Table 1 Characteristics of the studies included in the systematic review about Brazilian women with PCOS

\begin{tabular}{|c|c|c|c|c|c|}
\hline Name, Year & Region & PCOS criteria & Type of studies & N (PCOS/controls) & Age (PCOS/controls \\
\hline \multicolumn{6}{|l|}{ BMI-unmatched studies } \\
\hline Avila et al. 2014 [30] & Southeast & Rotterdam & Cross-sectional & $100 /-$ & $25.7 \pm 4.9 /-$ \\
\hline Azevedo et al. 2011 [23] & Northeast & Rotterdam & Cross-sectional & $113 / 242$ & $26.2 \pm 4.3 / 26.8 \pm 5.0$ \\
\hline Carvalho et al. 2017 [31] & Southeast & Rotterdam & Case-control & $86 / 86$ & $31.1 \pm 4.92 / 29.0 \pm 7.04$ \\
\hline Cerqueira et al. 2010 [24] & Northeast & Rotterdam & Cross-sectional & $56 / 54$ & $26.2 \pm 6.0 / 27.7 \pm 6.1$ \\
\hline Costa et al. 2012 [29] & Northeast & Rotterdam & Cross-sectional & $113 /-$ & $27.2 \pm 4.5 /-$ \\
\hline de Medeiros et al. 2014 [48] & Midwest & Rotterdam & Cross-sectional & 288/- & $26.9 \pm 5.5 /-$ \\
\hline Gabrielli et al. 2012 [26] & Northeast & Rotterdam & Cross-sectional & $73 / 725$ & $28.4 \pm 6.5 / 31.0 \pm 7.3^{*}$ \\
\hline Graff et al. 2017 [44] & South & Rotterdam & Case-control & $84 / 54$ & $23.5 \pm 6.3 / 26.2 \pm 6.5$ \\
\hline Kogure et al. 2012 [32] & Southeast & Rotterdam & Case-control & 20/19 & $27.8 \pm 5.0 / 27.9 \pm 5.2$ \\
\hline Maciel et al. 2014 [34] & Southeast & Rotterdam & Cross-sectional & $97 /-$ & $24.9 \pm 5.1 /-$ \\
\hline Melo et al. 2011ª [35] & Southeast & Rotterdam & Cross-sectional & $132 / 146$ & $26.6 \pm 5.1 / 28.9 \pm 0.5$ \\
\hline Oliveira et al. $2013^{b}[36]$ & Southeast & Rotterdam & Case-control & $42 / 18$ & $27.4 \pm 5.5 / 31.4 \pm 6.1$ \\
\hline Pedroso et al. 2012 [37] & Southeast & Rotterdam & Cross-sectional & $105 /-$ & $29 \pm 4.4 /-$ \\
\hline Pontes et al. 2012 [38] & Southeast & Rotterdam & Cross-sectional & 189/- & $24.9 \pm 5.2 /-$ \\
\hline Radavelli-Bagatini et al. 2013 [45] & South & Rotterdam & Case-control & $80 / 1500$ & $21.3 \pm 0.6 / 22.7 \pm 0.4$ \\
\hline Ramos et al. 2015 [46] & South & Rotterdam & Case-control & 199/99 & $22 \pm 6 / 25 \pm 7$ \\
\hline Santana et al. $2004^{c}[40]$ & Southeast & $\mathrm{NIH}$ & Cohort & $21 /-$ & $27.2 /-$ \\
\hline Soares et al. 2016 [27] & Northeast & Rotterdam & Cross-sectional & $22 /-$ & $26 \pm 6.0 /-$ \\
\hline Tavares et al. 2019 [28] & Northeast & Rotterdam & Cross-sectional & $111 /-$ & $18-39$ \\
\hline Wanderley et al., 2018 [49] & Midwest & Rotterdam & Cross-sectional & $83 /-$ & $28.79 \pm 5.85 /-$ \\
\hline Wiltgen et al. 2010 [43] & South & Rotterdam & Case-control & $195 / 25$ & $22.3 \pm 6.7 / 29.7 \pm 4.29^{*}$ \\
\hline Xavier et al. 2018 [42] & Southeast & Rotterdam & Case-control & $97 / 99$ & $30.5 \pm 5.1 / 29.8 \pm 7.1$ \\
\hline \multicolumn{6}{|l|}{ BMI-matched studies } \\
\hline Costa et al., 2008 [25] & Northeast & Rotterdam & Cross-sectional & $57 / 37$ & $25.5 \pm 5.3 / 26.6 \pm 5.4$ \\
\hline Lauria et al. 2013 [33] & Southeast & Rotterdam & Case-control & $40 / 36$ & $29(25-34) / 30(15-43)$ \\
\hline Rocha et al. 2011 [39] & Southeast & Rotterdam & Case-control & $142 / 31$ & $25.1 \pm 5.4 / 27.5 \pm 4$ \\
\hline Simões et al. 2017 [41] & Southeast & Rotterdam & Case-control & $10 / 10$ & $29.6 \pm 1.2 / 28.6 \pm 2.0$ \\
\hline Wiltgen et al. 2009 [47] & South & Rotterdam & Case-control & $51 / 44$ & $20.6 \pm 5.1 / 28.9 \pm 5.6^{*}$ \\
\hline \multicolumn{6}{|c|}{ a Data are from A phenotype PCOS vs controls } \\
\hline \multicolumn{6}{|c|}{ b Women included in the control group had similar complaints as the ones from the PCOS group, but did not fulfill the diagnostic criteria } \\
\hline \multicolumn{6}{|l|}{ c Data are from baseline } \\
\hline \multicolumn{6}{|c|}{ d Data are from A plus B phenotypes PCOS vs controls } \\
\hline${ }^{*} \mathrm{p}<0.05$ between groups & & & & & \\
\hline
\end{tabular}

between 2004 and 2019. Sample sizes ranged from 10 to 288 in PCOS groups and 10 to 1,500 in control groups.

\section{Qualitative data synthesis}

Table 1 summarizes the characteristics of studies on Brazilian populations. Seven studies were from the Northeast region [23-29], thirteen studies were from the Southeast [30-42], five studies were from the South [43-47], and two studies were from the Midwest region $[48,49]$. No data from the North region were available. All the studies used the Rotterdam criteria for diagnosis of PCOS, except by one [40]. In five studies, the control groups and PCOS participants were BMI-matched [25, $33,39,41,47]$. Ten out of 27 studies had no control group for comparison [27-30, 34, 37, 38, 40, 48, 49] and thus these studies were not included in the meta-analyses. Another one study was excluded from the meta-analyses because the control group included participants who were hirsute or had irregular menses [36]. Two studies in which data on PCOS participants were presented only as PCOS phenotypes $[35,43]$ and other two studies that did not present SD values for the variables of interest [29, 34] were equally excluded from the meta-analyses.

In Brazilian women with PCOS, BMI ranged from 24.2 to $31.9 \mathrm{~kg} / \mathrm{m}^{2}$ (Table 2). In studies without a BMImatched control group, most PCOS groups had higher BMI than controls [23, 24, 31, 35, 42-46]. The prevalence of obesity in Brazilian women with PCOS diagnosed by 
Table 2 Characteristics of Brazilian women with PCOS in the studies included in the systematic review

\begin{tabular}{|c|c|c|c|c|c|c|}
\hline $\begin{array}{l}\text { Name, } \\
\text { Year }\end{array}$ & BMI (PCOS/controls) & $\begin{array}{l}\text { Waist circumference } \\
(\mathrm{cm}) \text { (PCOS /controls) }\end{array}$ & $\begin{array}{l}\text { Blood pressure }(\mathrm{mmHg}) \\
\text { (PCOS/controls) }\end{array}$ & $\begin{array}{l}\text { Lipid profile (PCOS/ } \\
\text { controls) }\end{array}$ & $\begin{array}{l}\text { Glucose (mmol/L) } \\
\text { (PCOS/controls) }\end{array}$ & $\begin{array}{l}\text { HOMA-IR (PCOS/ } \\
\text { controls) }\end{array}$ \\
\hline \multicolumn{7}{|c|}{ BMI-unmatched studies } \\
\hline $\begin{array}{l}\text { Avila et al. } \\
2014[30]\end{array}$ & $30.6 \pm 9.3 /-$ & NA & NA & NA & NA & NA \\
\hline $\begin{array}{l}\text { Azevedo } \\
\text { et al. } \\
2011[23]\end{array}$ & $29.3 \pm 6.7 / 24.1 \pm 4.4^{*}$ & $91.2 \pm 15.7 / 77.1 \pm 9.6^{*}$ & $\begin{array}{l}\text { SBP: } 114.8 \pm 13.3 / 111.5 \pm 10.7^{*} \\
\text { DBP: } 72.6 \pm 10.7 / 72.1 \pm 10.3\end{array}$ & $\begin{array}{l}\mathrm{TC}(\mathrm{mmol} / \mathrm{L}): \\
4.82 \pm 1.09 / 4.57 \pm 1.19 \\
\mathrm{HDL}(\mathrm{mmol} / \mathrm{L}): \\
1.05 \pm 0.29 / 1.39 \pm 0.49^{*} \\
\mathrm{LDL}(\mathrm{mmol} / \mathrm{L}): \\
\mathrm{NA} \\
\mathrm{TGL}(\mathrm{mmol} / \mathrm{L}): \\
1.56 \pm 0.91 / 1.13 \pm 0.6 *\end{array}$ & $4.64 \pm 0.67 / 4.25 \pm 0.62^{*}$ & NA \\
\hline $\begin{array}{l}\text { Carvalho } \\
\text { et al. } \\
2017 \text { [31] }\end{array}$ & $30.1 \pm 5.4 / 23.2 \pm 4.23^{*}$ & $98.0(17.0) / 71.5(16.0)^{*}$ & NA & NA & $4.83 \pm 0.4 / 4.7 \pm 0.58$ & $3.54(4.8) / 1.68(1.6)^{*}$ \\
\hline $\begin{array}{l}\text { Cerqueira } \\
\text { et al. } \\
2010[24]\end{array}$ & $27.7 \pm 5.4 / 24 \pm 4.2^{*}$ & $84.5 \pm 11.3 / 78.9 \pm 10.0^{*}$ & $\begin{array}{l}\text { SBP: } 117.5 \pm 11.9 / 104.0 \pm 10.3^{*} \\
\text { DBP: } 77.7 \pm 9.8 / 68.7 \pm 8.1^{*}\end{array}$ & $\begin{array}{l}\text { TC }(\mathrm{mmol} / \mathrm{L}): \\
4.57 \pm 0.81 / 4.11 \pm 0.76^{*} \\
\mathrm{HDL}(\mathrm{mmol} / \mathrm{L}): \\
1.16 \pm 0.24 / 1.41 \pm 0.43^{*} \\
\mathrm{LDL}(\mathrm{mmol} / \mathrm{L}): \\
2.29 \pm 0.92 / 2.14 \pm 0.75 \\
\mathrm{TGL}(\mathrm{mmol} / \mathrm{L}): \\
1.3 \pm 0.78 / 0.94 \pm 0.43^{*}\end{array}$ & $4.88 \pm 0.68 / 4.68 \pm 0.41^{*}$ & $3.6 \pm 3.7 / 1.9 \pm 0.9^{*}$ \\
\hline $\begin{array}{l}\text { Costa et al. } \\
2012[29]\end{array}$ & $29.6 \pm 6.6 /-$ & NA & $\begin{array}{l}\text { SBP:115.5 } \pm 13.0 /- \\
\text { DBP:73.3 } \pm 10.3 /-\end{array}$ & $\begin{array}{l}\mathrm{TC}(\mathrm{mmol} / \mathrm{L}): \\
4.88 \pm 1.08 /- \\
\mathrm{HDL}(\mathrm{mmol} / \mathrm{L}): \\
01.14 \pm 0.28 /- \\
\mathrm{LDL}(\mathrm{mmol} / \mathrm{L}) \\
3.11 \pm 1.07 /- \\
\mathrm{TGL}(\mathrm{mmol} / \mathrm{L}): \\
\quad 1.54 \pm 0.81 /-\end{array}$ & $4.62 \pm 0.68 /-$ & NA \\
\hline $\begin{array}{l}\text { de Medei- } \\
\text { ros et al. } \\
2014[48]\end{array}$ & $29.9 \pm 7.0 /-$ & $88.0 \pm 16.3 /-$ & NA & NA & $5.11 \pm 0.78 /-$ & $1.93 \pm 1.21 /-$ \\
\hline $\begin{array}{l}\text { Gabrielli } \\
\text { et al. } \\
2012[26]\end{array}$ & $\begin{array}{l}24.2(17.7-30.7) / 24.1 \\
\quad(18.1-30.1)\end{array}$ & $74(56-92) / 75(60-90)$ & $\begin{array}{l}\text { SBP: } 119(99-139) / 122 \\
\quad(102-142) \\
\text { DBP: } 74(60-88) / 74(59.5-89.5)\end{array}$ & NA & NA & NA \\
\hline $\begin{array}{l}\text { Graff et al. } \\
2017[44]\end{array}$ & $29.4 \pm 6.4 / 27.2 \pm 5.8^{*}$ & $86.6 \pm 14.1 / 83.6 \pm 12.3$ & $\begin{array}{l}\text { SBP:118.2 } \pm 13.0 / 112.4 \pm 11.1^{*} \\
\text { DBP:77.4 } \pm 9.9 / 72.8 \pm 10.0^{*}\end{array}$ & $\begin{array}{l}\text { TC }(\mathrm{mmol} / \mathrm{L}): \\
4.49 \pm 0.89 / 4.5 \pm 0.79 \\
\mathrm{HDL}(\mathrm{mmol} / \mathrm{L}): \\
1.18 \pm 0.31 / 1.31 \pm 0.28^{*} \\
\mathrm{LDL}(\mathrm{mmol} / \mathrm{L}): \\
2.75 \pm 0.71 / 2.72 \pm 0.63 \\
\mathrm{TGL}(\mathrm{mmol} / \mathrm{L}): \\
0.99(0.69-1.53) \\
\quad / 0.87(0.6-1.16)\end{array}$ & $4.85 \pm 0.47 / 4.82 \pm 0.44$ & $\begin{array}{l}3.4(1.8-4.7) / 2.1(1.5- \\
2.8)^{*}\end{array}$ \\
\hline $\begin{array}{l}\text { Kogure } \\
\text { et al. } \\
2012[32]\end{array}$ & $28.7 \pm 4.4 / 27.1 \pm 5.1$ & NA & NA & $\begin{array}{l}\mathrm{TC}(\mathrm{mmol} / \mathrm{L}): \\
5.42 \pm 1.24 / 5.47 \pm 1.19 \\
\mathrm{HDL}(\mathrm{mmol} / \mathrm{L}): \\
1.38 \pm 0.31 / 1.52 \pm 0.28 \\
\mathrm{LDL}(\mathrm{mmol} / \mathrm{L}): \\
\quad 3.29 \pm 0.92 / 3.4 \pm 0.96 \\
\mathrm{TGL}(\mathrm{mmol} / \mathrm{L}): \\
\quad 1.65 \pm 0.93 / 1.19 \pm 0.75^{*}\end{array}$ & $5.82 \pm 0.99 / 5.73 \pm 1.09$ & $2.3 \pm 2.3 / 1.6 \pm 0.8^{*}$ \\
\hline $\begin{array}{l}\text { Maciel et al. } \\
2014[34]\end{array}$ & $29.6 \pm 6.9 /-$ & $90.1 \pm 15.2 /-$ & NA & $\begin{array}{l}\mathrm{TC}(\mathrm{mmol} / \mathrm{L}): 4.42 \pm 0.82 /- \\
\mathrm{HDL}(\mathrm{mmol} / \mathrm{L}): \\
\quad 1.3 \pm 0.36 /- \\
\mathrm{LDL}(\mathrm{mmol} / \mathrm{L}): \\
\quad 2.55 \pm 0.67 /- \\
\mathrm{TGL}(\mathrm{mmol} / \mathrm{L}): 1.3 \pm 0.7 /-\end{array}$ & $4.95 \pm 0.57 /-$ & $3.8 \pm 3.3 /-$ \\
\hline
\end{tabular}


Table 2 (continued)

\begin{tabular}{|c|c|c|c|c|c|c|}
\hline $\begin{array}{l}\text { Name, } \\
\text { Year }\end{array}$ & BMI (PCOS/controls) & $\begin{array}{l}\text { Waist circumference } \\
\text { (cm) (PCOS /controls) }\end{array}$ & $\begin{array}{l}\text { Blood pressure }(\mathrm{mmHg}) \\
\text { (PCOS/controls) }\end{array}$ & $\begin{array}{l}\text { Lipid profile (PCOS/ } \\
\text { controls) }\end{array}$ & $\begin{array}{l}\text { Glucose (mmol/L) } \\
\text { (PCOS/controls) }\end{array}$ & $\begin{array}{l}\text { HOMA-IR (PCOS/ } \\
\text { controls) }\end{array}$ \\
\hline $\begin{array}{l}\text { Melo et al. } \\
2011^{\text {a }} \\
{[35]}\end{array}$ & $31.3 \pm 8.7 / 24.4 \pm 4.9^{*}$ & $98.6 \pm 17.7 / 84.8 \pm 12.4$ & $\begin{array}{l}\text { SBP: } \\
121.5 \pm 15.2 / 111.5 \pm 10.8^{*} \\
\text { DBP: } \\
78 \pm 10.4 / 71.7 \pm 8.2^{*}\end{array}$ & $\begin{array}{l}\mathrm{TC}(\mathrm{mmol} / \mathrm{L}): \\
4.76 \pm 1.04 / 4.93 \pm 0.84 \\
\mathrm{HDL}(\mathrm{mmol} / \mathrm{L}): \\
\quad 1.16 \pm 0.27 / 1.41 \pm 0.28^{*} \\
\mathrm{LDL}(\mathrm{mmol} / \mathrm{L}): \\
\quad 2.9 \pm 0.92 / 2.82 \pm 0.72 \\
\mathrm{TGL}(\mathrm{mmol} / \mathrm{L}): \\
\quad 1.49 \pm 0.82 / 0.95 \pm 0.44^{*}\end{array}$ & $5.17 \pm 1.41 / 4.58 \pm 0.49^{*}$ & $4.5 \pm 4.9 / 1.5 \pm 1.1^{*}$ \\
\hline $\begin{array}{l}\text { Oliveira } \\
\text { et al. } \\
2013^{b} \\
{[36]}\end{array}$ & $30.2 \pm 6.5 / 27.1 \pm 6.2$ & NA & $\begin{array}{l}\text { SBP: } \\
111.8 \pm 12.0 / 107.3 \pm 15.0 \\
\text { DBP: } \\
70.2 \pm 9.0 / 71 \pm 13.5\end{array}$ & $\begin{array}{l}\mathrm{TC}(\mathrm{mmol} / \mathrm{L}): \mathrm{NA} \\
\mathrm{HDL}(\mathrm{mmol} / \mathrm{L}): \\
\quad 1.3 \pm 0.38 / 1.49 \pm 0.35 \\
\mathrm{LDL}(\mathrm{mmol} / \mathrm{L}): \mathrm{NA} \\
\mathrm{TGL}(\mathrm{mmol} / \mathrm{L}): \\
\quad 1.32 \pm 0.91 / 1.31 \pm 0.74\end{array}$ & $4.82 \pm 0.97 / 4.54 \pm 0.47$ & $4.4 \pm 6.8 / 2.1 \pm 1.1$ \\
\hline $\begin{array}{l}\text { Pedroso } \\
\text { et al. } \\
2012 \text { [37] }\end{array}$ & $31.9 \pm 8.2 /-$ & $99 \pm 16.6)$ & $\begin{array}{l}\text { SBP: } \\
122.5 \pm 18.7 /- \\
\text { DBP: } \\
79 \pm 11 /-\end{array}$ & $\begin{array}{l}\mathrm{TC}(\mathrm{mmol} / \mathrm{L}): 4.86 \pm 1.01 /- \\
\mathrm{HDL}(\mathrm{mmol} / \mathrm{L}): \\
1.22 \pm 0.32 /- \\
\mathrm{LDL}(\mathrm{mmol} / \mathrm{L}): \\
2.94 \pm 0.87 /- \\
\mathrm{TGL}(\mathrm{mmol} / \mathrm{L}): \\
1.48 \pm 0.84 /-\end{array}$ & $5.04 \pm 1.08 /-$ & NA \\
\hline $\begin{array}{c}\text { Pontes et al. } \\
2012 \text { [38] }\end{array}$ & $31.8 \pm 7.6 /-$ & $92.2 \pm 16.0$ & $\begin{array}{l}\text { SBP: } 116.3 \pm 14.4 /- \\
\text { DBP: } 75.1 \pm 10.4 /-\end{array}$ & $\begin{array}{l}\text { TC }(\mathrm{mmol} / \mathrm{L}): 4.73 \pm 0.9 /- \\
\mathrm{HDL}(\mathrm{mmol} / \mathrm{L}): \\
\quad 1.21 \pm 0.33 /- \\
\mathrm{LDL}(\mathrm{mmol} / \mathrm{L}): \\
2.90 \pm 0.77 /- \\
\text { TGL }(\mathrm{mmol} / \mathrm{L}): \\
1.43 \pm 0.91 /-\end{array}$ & $4.87 \pm 0.38 /-$ & NA \\
\hline $\begin{array}{l}\text { Radavelli- } \\
\text { Bagatini } \\
\text { et al. } \\
2013 \text { [45] }\end{array}$ & $31.0 \pm 7.9 / 23.4 \pm 4.6^{*}$ & $92.2 \pm 18.8 / 74.5 \pm 10.2^{*}$ & $\begin{array}{l}\text { SBP: } 124.6 \pm 19.9 / 111.5 \pm 13.0^{*} \\
\text { DBP: } 79.2 \pm 12.5 / 71.8 \pm 10.6^{*}\end{array}$ & $\begin{array}{l}\mathrm{TC}(\mathrm{mmol} / \mathrm{L}): \\
4.81 \pm 1.16 / 4.24 \pm 0.9^{*} \\
\mathrm{HDL}(\mathrm{mmol} / \mathrm{L}): \\
1.32 \pm 0.28 / 1.52 \pm 0.36^{*} \\
\mathrm{LDL}(\mathrm{mmol} / \mathrm{L}): \\
2.95 \pm 0.98 / 2.09 \pm 0.72^{*} \\
\mathrm{TGL}(\mathrm{mmol} / \mathrm{L}): \mathrm{NA}\end{array}$ & NA & NA \\
\hline $\begin{array}{l}\text { Ramos et al. } \\
2015 \text { [46] }\end{array}$ & $29.6 \pm 6.4 / 27.6 \pm 6.0^{*}$ & $89.2 \pm 15.0 / 78.1 \pm 11.5^{*}$ & NA & NA & $4.93 \pm 0.68 / 4.91 \pm 0.42$ & NA \\
\hline $\begin{array}{l}\text { Santana } \\
\text { et al. } \\
2004^{c} \\
{[40]}\end{array}$ & $29.18 \pm 7.78 /-$ & $89.36 \pm 15.23 /-$ & NA & $\begin{array}{l}\mathrm{TC}(\mathrm{mmol} / \mathrm{L}): 4.71 \pm 0.98 /- \\
\mathrm{HDL}(\mathrm{mmol} / \mathrm{L}): \\
1.01 \pm 0.2 /- \\
\mathrm{LDL}(\mathrm{mmol} / \mathrm{L}): \\
3.10 \pm 0.86 /- \\
\mathrm{TGL}(\mathrm{mmol} / \mathrm{L}): \\
1.26 \pm 0.67 /-\end{array}$ & $4.48 \pm 0.59 /-$ & NA \\
\hline $\begin{array}{l}\text { Soares et al. } \\
2016 \text { [27] }\end{array}$ & $29.8 \pm 6.1 /-$ & $95.4 \pm 15.8 /-$ & NA & $\begin{array}{l}\mathrm{TC}(\mathrm{mmol} / \mathrm{L}): \mathrm{NA} \\
\mathrm{HDL}(\mathrm{mmol} / \mathrm{L}): \\
1.13 \pm 0.35 /- \\
\mathrm{LDL}(\mathrm{mmol} / \mathrm{L}): 2.3(1.81- \\
2.69) /- \\
\mathrm{TGL}(\mathrm{mmol} / \mathrm{L}): 1.39(0.71- \\
\quad 1.85) /-\end{array}$ & $4.38 \pm 0.53 /-$ & $1.9(1.3-3.3) /-$ \\
\hline $\begin{array}{l}\text { Wanderley } \\
\text { et al. } \\
2018 \text { [49] }\end{array}$ & $29.9 \pm 5.28 /-$ & $92.15 \pm 10.72 /-$ & $\begin{array}{l}\text { SBP: } 123.15 \pm 18.38 /- \\
\text { DBP: } 79.13 \pm 11.00 /-\end{array}$ & $\begin{array}{l}\mathrm{TC}(\mathrm{mmol} / \mathrm{L}): 4.73 \pm 0.9 /- \\
\mathrm{HDL}(\mathrm{mmol} / \mathrm{L}): \\
1.28 \pm 0.33 /- \\
\mathrm{LDL}(\mathrm{mmol} / \mathrm{L}): \\
3.03 \pm 0.85 /- \\
\mathrm{TGL}(\mathrm{mmol} / \mathrm{L}): \\
1.25 \pm 0.67 /-\end{array}$ & $4.87 \pm 0.36 /-$ & NA \\
\hline
\end{tabular}


Table 2 (continued)

\begin{tabular}{|c|c|c|c|c|c|c|}
\hline $\begin{array}{l}\text { Name, } \\
\text { Year }\end{array}$ & BMI (PCOS/controls) & $\begin{array}{l}\text { Waist circumference } \\
\text { (cm) (PCOS /controls) }\end{array}$ & $\begin{array}{l}\text { Blood pressure }(\mathrm{mmHg}) \\
\text { (PCOS/controls) }\end{array}$ & $\begin{array}{l}\text { Lipid profile (PCOS/ } \\
\text { controls) }\end{array}$ & $\begin{array}{l}\text { Glucose (mmol/L) } \\
\text { (PCOS/controls) }\end{array}$ & $\begin{array}{l}\text { HOMA-IR (PCOS/ } \\
\text { controls) }\end{array}$ \\
\hline $\begin{array}{l}\text { Wiltgen } \\
\text { et al. } \\
2010^{d} \\
{[43]}\end{array}$ & $31 \pm 7.98 / 26.97 \pm 3.6^{*}$ & $\begin{array}{l}93.79 \pm 18.81 / \\
\quad 79.83 \pm 8.37^{*}\end{array}$ & $\begin{array}{l}\text { SBP: } 123.1 \pm 16.9 / 115.2 \pm 9.5^{*} \\
\text { DBP: } 78.9 \pm 12.3 / 73.6 \pm 8.3\end{array}$ & $\begin{array}{l}\mathrm{TC}(\mathrm{mmol} / \mathrm{L}): \\
\quad 4.72 \pm 1.13 / 4.27 \pm 0.95 \\
\mathrm{HDL}(\mathrm{mmol} / \mathrm{L}): \\
1.25 \pm 0.29 / 1.42 \pm 0.35 \\
\mathrm{LDL}(\mathrm{mmol} / \mathrm{L}): 2.85 \pm 0.96 / \\
\quad 2.47 \pm 0.81 \\
\mathrm{TGL}(\mathrm{mmol} / \mathrm{L}): 1.12 \\
\quad(0.76-1.6) / 0.68(0.47 \\
-1.05)^{*}\end{array}$ & $5.02 \pm 1.19 / 4.92 \pm 0.45$ & $\begin{array}{l}4.53(2.6-7.7) / 2.14 \\
\quad(1.4-3.1)^{*}\end{array}$ \\
\hline $\begin{array}{l}\text { Xavier et al. } \\
2018 \text { [42] }\end{array}$ & $28.8 \pm 8.1 / 22.9 \pm 5.9^{*}$ & $97.0(18.0) / 82.4(20.0)^{*}$ & NA & $\begin{array}{l}\mathrm{TC}(\mathrm{mmol} / \mathrm{L}): 4.92 \pm 0.94 / \\
4.55 \pm 0.83^{*} \\
\mathrm{HDL}(\mathrm{mmol} / \mathrm{L}): 1.19(0.49) \\
/ 1.39(0.49)^{*} \\
\mathrm{LDL}(\mathrm{mmol} / \mathrm{L}): \\
2.97 \pm 0.82 / 2.58 \pm 0.72^{*} \\
\mathrm{TGL}(\mathrm{mmol} / \mathrm{L}): 1.11(0.97) \\
/ 0.93(0.44)^{*}\end{array}$ & $6.95 \pm 11.2 / 5.78 \pm 7.14$ & $2.8(1.8) / 1.59(1.2)^{*}$ \\
\hline \multicolumn{7}{|c|}{ BMI-matched studies } \\
\hline $\begin{array}{l}\text { Costa et al. } \\
2008[25]\end{array}$ & $27.6 \pm 5.8 / 26.7 \pm 4.9$ & $87.8 \pm 14.3 / 83.6 \pm 10.1$ & $\begin{array}{l}\text { SBP: } 118.9 \pm 15.2 / 113.8 \pm 10.9 \\
\text { DBP: } 79.9 \pm 8.9 / 73.4 \pm 10.2^{*}\end{array}$ & $\begin{array}{l}\mathrm{TC}(\mathrm{mmol} / \mathrm{L}): \\
4.37 \pm 0.62 / 4.11 \pm 0.83 \\
\mathrm{HDL}(\mathrm{mmol} / \mathrm{L}): \\
1.23 \pm 0.34 / 1.54 \pm 0.27^{*} \\
\mathrm{LDL}(\mathrm{mmol} / \mathrm{L}): \\
2.56 \pm 0.65 / 2.21 \pm 0.72^{*} \\
\mathrm{TGL}(\mathrm{mmol} / \mathrm{L}): \\
\quad 1.16 \pm 0.55 / 1.06 \pm 0.68\end{array}$ & & \\
\hline $\begin{array}{l}\text { Lauria et al. } \\
\qquad 2013 \text { [33] }\end{array}$ & $27.64 \pm 5.43 / 25.99 \pm 5.51$ & $91(83-101) / 94(83-103)$ & $\begin{array}{l}\text { SBP: } \\
120(110-120) / 120(110-120) \\
\text { DBP: } \\
80(70-80) / 80(70-80)\end{array}$ & $\begin{array}{l}\mathrm{TC}(\mathrm{mmol} / \mathrm{L}): \\
0000000000000004.37 \\
(3.67-4.76) / 3.67 \\
(3.21-4.34)^{*} \\
\mathrm{HDL}(\mathrm{mmol} / \mathrm{L}): 1.11(0.88- \\
1.29) / 1.01(0.85-1.14) \\
\mathrm{LDL}(\mathrm{mmol} / \mathrm{L}): 2.77(2.3- \\
3.26) / 2.25(1.76-2.87)^{*} \\
\mathrm{TGL}(\mathrm{mmol} / \mathrm{L}): 0.91(0.73- \\
1.22) / 0.8(0.65-1.06)\end{array}$ & & \\
\hline $\begin{array}{l}\text { Rocha et al. } \\
2011 \text { [39] }\end{array}$ & $29.1 \pm 6.17 / 27.4 \pm 6.9$ & NA & NA & $\begin{array}{l}\mathrm{TC}(\mathrm{mmol} / \mathrm{L}): \\
\quad 4.68 \pm 0.78 / 4.28 \pm 0.4 \\
\mathrm{HDL}(\mathrm{mmol} / \mathrm{L}): \\
\quad 1.23 \pm 0.45 / 1.51 \pm 0.2^{*} \\
\mathrm{LDL}(\mathrm{mmol} / \mathrm{L}): \\
\quad 2.87 \pm 0.65 / 2.63 \pm 0.58 \\
\mathrm{TGL}(\mathrm{mmol} / \mathrm{L}): \\
\quad 1.34 \pm 0.74 / 1.32 \pm 0.4\end{array}$ & & \\
\hline $\begin{array}{l}\text { Simões } \\
\text { et al. } \\
2017[41]\end{array}$ & $28.0 \pm 2.4 / 27.4 \pm 2.4$ & NA & NA & NA & & \\
\hline $\begin{array}{l}\text { Wiltgen } \\
\text { et al. } \\
2009 \text { [47] }\end{array}$ & $29.5 \pm 7.5 / 29.4 \pm 5.4$ & $90.6 \pm 16.1 / 85.5 \pm 11.6^{*}$ & NA & $\begin{array}{l}\mathrm{TC}(\mathrm{mmol} / \mathrm{L}): 4.85 \pm 1.11 / \\
4.2 \pm 0.8^{*} \\
\mathrm{HDL}(\mathrm{mmol} / \mathrm{L}): 1.35 \pm 0.27 \\
/ 1.32 \pm 0.3 \\
\mathrm{LDL}(\mathrm{mmol} / \mathrm{L}) \\
3.07 \pm 0.97 / 2.46 \pm 0.69^{*} \\
\mathrm{TGL}(\mathrm{mmol} / \mathrm{L}): 1.1(0.77- \\
1.48) / 0.73(0.54-1.21)^{*}\end{array}$ & & \\
\hline
\end{tabular}

a Data are from A phenotype PCOS vs controls

b Women included in the control group had similar complaints as the ones from the PCOS group, but did not fulfill the diagnostic criteria

c Data are from baseline

d Data are from A plus B phenotypes PCOS vs controls

${ }^{*} p<0.05$ between the groups. Continuous metabolic variables are not available from Tavares et al. 2019 [24] 
Rotterdam criteria was reported in eight studies [26, 28, 34, 39, 42, 46, 48, 49]. Except for one study with nonselected women from primary healthcare services [26] in which the prevalence of obesity was similar to that of the general Brazilian female population [50] in the other seven studies the prevalence of obesity ranged from 31.6 to 56.6 in the Midwest, Southeast, and South, showing quite higher values than those expected for the Brazilian female population (17.9\% for women aged $25-34$ years) [50] (Table 3).

Table 2 also presents WC, blood pressure, glucose, and lipid profile of Brazilian women with PCOS and control populations. Only three studies $[26,37,44]$ did not observe a larger WC in PCOS participants vs. controls. Thirteen studies had no data on WC or a control group for comparison.

Eleven Brazilian studies reported blood pressure data in PCOS and control groups [23-26, 33, 35, 36, 43-46]. Of these studies, eight showed a higher systolic (SBP) or diastolic blood pressure (DBP) in PCOS [23-25, 35, 43-46].

Fasting glucose was measured in 23 studies [23-25, 27, 29, 31-44, 46-49] (Table 2). Glucose levels ranged from 4.38 to $6.95 \mathrm{mmol} / \mathrm{L}$ in Brazilian women with PCOS and from 4.25 to 5.78 in controls. In three studies [23, 24, 37] PCOS groups had higher glucose levels than control groups. Eleven studies had no data on glucose levels or a control group for comparison.

Impaired fasting glucose (IFG, fasting glucose between 5.6 and $6.9 \mathrm{mmol} / \mathrm{L}$ ) [46] and/or impaired glucose tolerance (IGT, glucose levels between 7.8 and $11.1 \mathrm{mmol} / \mathrm{L}$ at $120 \mathrm{~min}$ after the oGTT) [43] was found in only two studies from the South region (9.7 and 11.3\%) and in one from the Northeast (7.2\% [28]). Only one study (Table 3 ) reported prevalence of type 2 diabetes in Brazilian women with PCOS. Out of 195 patients, $3.6 \%$ were diabetic.

HOMA-IR, a marker of insulin resistance, was available in 14 studies, and twelve studies also compared HOMAIR values in PCOS and controls. In ten studies [24, 25, 31, 32, 35, 39, 42-44, 47] HOMA-IR was higher in women with PCOS than in controls (Table 2). Metabolic syndrome was evaluated in Brazilian women with PCOS in the Northeast, Southeast, and South and showed a homogeneous prevalence among the regions, ranging between 24.6 and 42.9\% [28-30, 34, 37, 43, 46] (Table 3).

Regarding lipid profile, 19 studies on Brazilian women with PCOS showed TGL levels ranging from 0.91 to $1.65 \mathrm{mmol} / \mathrm{L}$, and in 12 of them values for control groups ranged from 0.68 to $1.32 \mathrm{mmol} / \mathrm{L}$ (Table 2). TGL levels were higher in PCOS than controls in seven out of these 12 studies [23, 24, 32, 35, 42, 43, 47]. Twenty-one Brazilian studies assessed HDL cholesterol levels, and 14 of them compared HDL values in PCOS (1.01$1.38 \mathrm{mmol} / \mathrm{L})$ vs. controls $(1.01-1.54 \mathrm{mmol} / \mathrm{L})[23-25$, $32,33,35,36,39,42-47$ ] (Table 2). In nine of these studies, HDL was significantly lower in PCOS than in controls [23-25, 35, 39, 42, 44-46]. LDL levels ranged from 2.29 to $3.29 \mathrm{mmol} / \mathrm{L}$ in PCOS women from 18 Brazilian studies and from 2.09 to $3.4 \mathrm{mmol} / \mathrm{L}$ in controls from 11 studies (Table 2). Five studies [25, 33, 42, 45, 47] reported

Table 3 Prevalence of obesity, dyslipidemia, metabolic syndrome, prediabetes and diabetes 2 in Brazilian women with PCOS

\begin{tabular}{|c|c|c|c|c|c|c|c|c|}
\hline Study & $\mathrm{N}$ & Region & Obesity (\%) & $\begin{array}{l}\text { Dyslipidemia (\%) } \\
\downarrow \mathrm{HDL}^{\mathrm{a}}\end{array}$ & $\uparrow \mathrm{TGL}^{\mathbf{b}}$ & MetS (\%) & $\begin{array}{l}\text { Prediabetes }{ }^{c} \\
(\%)\end{array}$ & $\begin{array}{l}\text { Type } 2 \\
\text { Diabetes } \\
(\%)\end{array}$ \\
\hline Avila et al. 2014 [30] & 100 & Southeast & & & & 36 & & \\
\hline Costa et al. 2012 [29] & 113 & Northeast & & 76.1 & 33.6 & 31 & & \\
\hline de Medeiroset al. 2014 [48] & 288 & Midwest & 44.3 & & & & & \\
\hline Gabrielli et al. 2012 [26] & 73 & Northeast & 13.7 & & & & & \\
\hline Maciel et al. 201434 & 97 & Southeast & 42.3 & 52.6 & 22.7 & 26.8 & & \\
\hline Pedroso et al. 2012 [37] ${ }^{d}$ & 105 & Southeast & & 68.6 & 5.3 & 42.9 & & \\
\hline Ramos et al. 2015 [46] & 199 & South & & 56.6 & 17.8 & 24.6 & 9.7 & \\
\hline Rocha et al. 2011 [28] & 142 & Southeast & 31.6 & 57.6 & 28.3 & & & \\
\hline Tavares et al. 2019 [28] & 111 & Northeast & 44.1 & 54.1 & 35.1 & 33.6 & 7.2 & \\
\hline Wanderley et al. 2018 [49] & 83 & Midwest & 56.62 & & & & & \\
\hline Wiltgen et al. 2010 [43] & 195 & South & 52.5 & 58.8 & 22.9 & 31.3 & 11.3 & 3.6 \\
\hline Xavier,et al., 2018 [43] & 97 & Southeast & 42.3 & & & & & \\
\hline
\end{tabular}

Rotterdam criteria for all studies

a Lower HDL: $\leq 50$

b Higher TGL: $\geq 150$

c Prediabetes: IFG and/or IGT

d only data of adult women with PCOS were extracted 
LDL to be higher in PCOS. Eighteen studies with Brazilian women with PCOS assessed TC levels and 12 compared PCOS with controls, with mean values ranging from 4.37 to $5.42 \mathrm{mmol} / \mathrm{L}$ in PCOS and from 4.11 to 5.47 in controls (Table 2). Five studies showed higher TC levels in the PCOS group compared with the control group $[24,33,42,45,47]$. Seven studies assessed the prevalence of dyslipidemia in the Northeast, Southeast, and South and showed homogeneous values among the regions, for both lower HDL-cholesterol (ranging between 52.6 and $76.1 \%$ ) and higher triglycerides (from 5.3 to $35 \%$ ).

Three studies evaluating referral populations assessed the prevalence of PCOS phenotypes [28, 35, 43]. Phenotypes $\mathrm{A}+\mathrm{B}$ were more prevalent in these studies $(66.4$, 81 and $65.8 \%$, respectively).

\section{Quantitative data synthesis and meta-analysis}

Of 27 studies, 12 articles meeting eligibility criteria were included in the meta-analysis [23-25, 31, 32, 39, 41, 42, 44-47], for a total of 995 PCOS and 2,275 control women. All used the Rotterdam criteria to define PCOS. NOS score was 9 in seven studies, 8 in two, 7 in two, and 6 in another one (Table 4).

\section{BMI}

Only BMI-unmatched studies were considered for analysis. Data from seven studies were analyzed [23, 24, 31, 32, 44-46] including 638 PCOS and 2,054 controls. The PCOS group had higher BMI levels than controls (SMD 0.67, 95\% CI, 0.29, 1.05). Between-study heterogeneity was high $\left(I^{2}=91 \%, p<0.001\right)$ (Fig. 2a).

Table 4 Newcastle-Ottawa quality (NOS) assessment scale for studies included in the meta-analysis

\begin{tabular}{lllll}
\hline Author & Year & Selection & Comparability & Exposure \\
\hline Azevedo (23) & 2011 & $* * * *$ & $* *$ & $* * *$ \\
Carvalho (31) & 2017 & $* * * *$ & $* *$ & $* * *$ \\
Cerqueira (24) & 2010 & $* *$ & $*$ & $* * *$ \\
Costa (25) & 2008 & $* * *$ & $*$ & $* * *$ \\
Graff (44) & 2017 & $* * * *$ & $*$ & $* * *$ \\
Kogure (32) & 2012 & $* * * *$ & $* *$ & $* * *$ \\
Radavelli- Bagatini (45) & 2013 & $* * * *$ & $*$ & $* * *$ \\
Ramos (46) & 2015 & $* * * *$ & $* *$ & $* * *$ \\
Rocha (39) & 2011 & $* * * *$ & $* *$ & $* * *$ \\
Simões (41) & 2017 & $* * * *$ & $* *$ & $* * *$ \\
Wiltgen (47) & 2009 & $* * * *$ & $* *$ & $* * *$ \\
Xavier (42) & 2018 & $* * *$ & $*$ & $* * *$ \\
\hline
\end{tabular}

Quality of selection (minimum 1-maximum 4 stars); Comparability (minimum 0-maximum 2 stars); Exposure (minimum 1-maximum 3 stars)

\section{Waist circumference}

As for BMI, only BMI-unmatched studies were considered for analysis of waist circumference. Five studies [23, 24, 44-46] with 532 PCOS and 1,949 control participants were considered. The PCOS group had higher waist circumference vs. the control group (SMD 0.88, 95\% CI $0.40,1.37)$. Between-study heterogeneity was high $\left(\mathrm{I}^{2}=93 \% \mathrm{p}=0.001\right)$ (Fig. 2b).

\section{Blood pressure}

Five studies entered the meta-analysis of blood pressure [23-25, 44, 45] with 390 PCOS and 1887 control participants. Higher SBP (SMD 0.66, 95\% CI 0.30, 1.01) and DBP levels (SMD 0.55, 95\% CI 0.24, 0.87) were observed in women with PCOS than in controls $\left(\mathrm{I}^{2}=83 \%, \mathrm{p}<0.001\right.$ for SBP and $\mathrm{I}^{2}=81 \%, \mathrm{p}=0.0001$ for DBP) (Fig. $2 \mathrm{c}$ and $\mathrm{d}$ ).

\section{Glucose}

Ten studies were included for glucose meta-analysis [23$25,32,39,41,42,44,46,47]$ including 829 PCOS and 689 control participants. Glucose levels were higher in the PCOS group (SMD 0.21, 95\% CI 0.04, 0.38) when compared with controls, with moderate between-study heterogeneity $\left(\mathrm{I}^{2}=54.8 \%, \mathrm{p}=0.011\right)$ (Fig. 3a).

\section{HOMA-IR}

HOMA-IR was analyzed in four studies [23, 25, 32, 39], for a total of 275 PCOS and 141 controls. HOMA-IR was higher in PCOS vs. controls (SMD 0.78, 95\% CI 0.52, $1.04)$, with low between-study heterogeneity $\left(\mathrm{I}^{2}=26 \%\right.$, $\mathrm{p}=0.23)$ (Fig. 3b).

\section{Lipids}

Data from five studies were available for TGL analysis [23-25, 32, 39], including 388 PCOS and 383 control participants. TGL levels were higher in the PCOS group (SMD of $0.39,95 \%$ CI $0.14,0.64$ ), with moderate between-study heterogeneity $\left(\mathrm{I}^{2}=63 \%, \mathrm{p}=0.079\right)$ (Fig. 4a). Eight studies were included in the HDL metaanalysis [23-25, 32, 39, 44, 45, 47], with 603 PCOS and 1,981 controls. HDL levels were lower in the PCOS group (SMD - 0.56, 95\% CI - 0.78, - 0.34) when compared with controls. Between-study heterogeneity was moderate $\left(\mathrm{I}^{2}=68 \%, \mathrm{p}=0.006\right)$ (Fig. $\left.4 \mathrm{~b}\right) . \mathrm{LDL}$ data were included from eight studies [24, 25, 32, 39, 42, 44, $45,47]$ with 587 PCOS and 1,838 controls. LDL levels were higher in PCOS (SMD 0.45 95\% CI 0.17, 0.74). Between-study heterogeneity was high $\left(\mathrm{I}^{2}=80.31 \%\right.$, $\mathrm{p}=<0.0001$ ) (Fig. 4c). Nine studies compared total cholesterol levels in PCOS and controls [23-25, 32, $39,42,44,45,47]$ for a total of 700 PCOS and 2080 


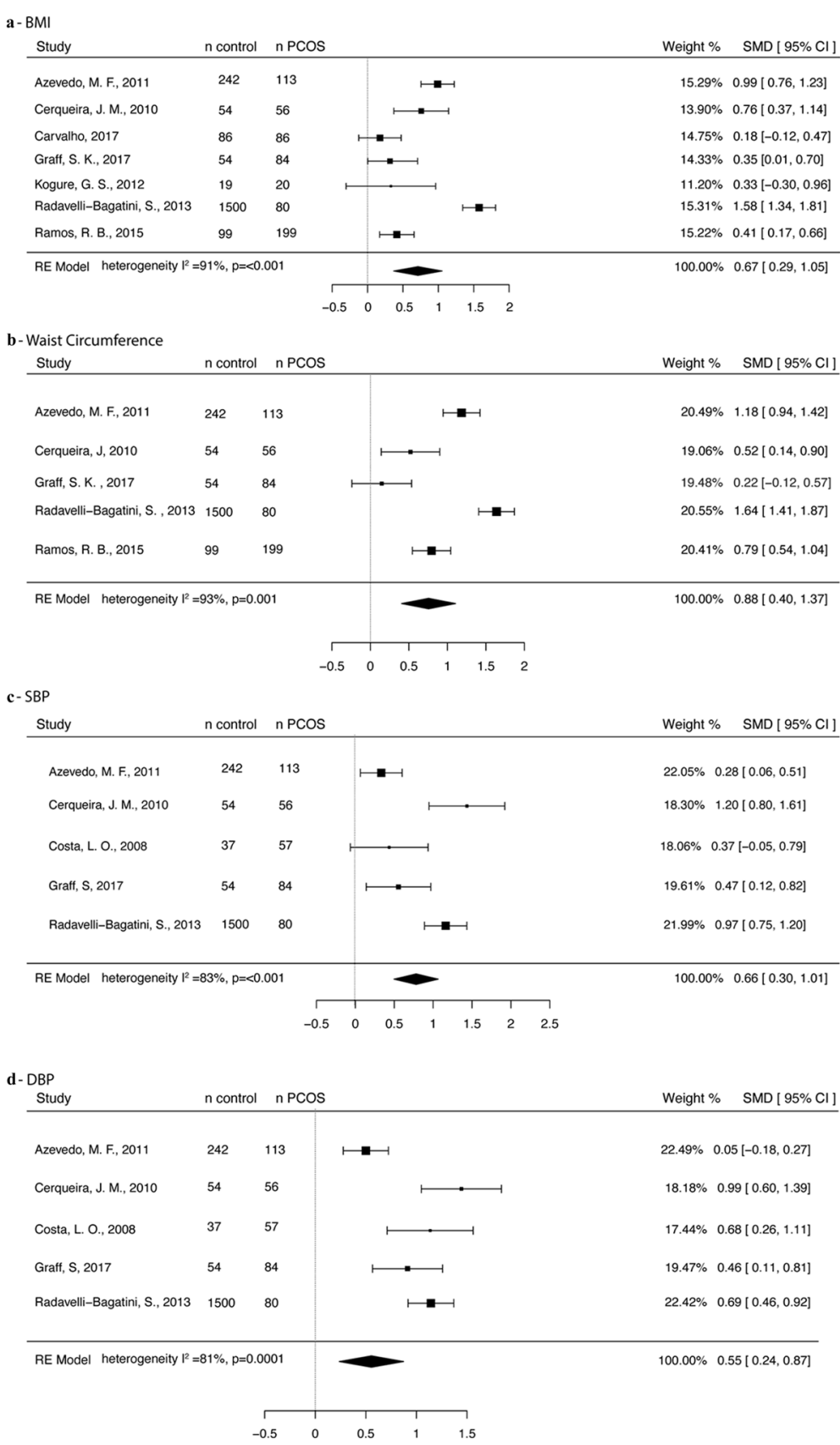

Fig. 2 Forest plot showing a BMI, b waist circumference, c systolic blood pressure and $\mathbf{d}$ diastolic blood pressure

controls. TC levels were higher in PCOS (SMD 0.40 $95 \%$ CI $0.24,0.57$ ) than control participants, with moderate between-study heterogeneity $\left(\mathrm{I}^{2}=52 \%, \mathrm{p}=0.02\right)$ (Fig. 4d).

Publication bias may have occurred in comparisons of PCOS vs. controls in LDL analysis (Fig. 5i). Conversely, no publication bias was detected in any other comparisons ( $p>=0.10$; Fig. 5a-h and j).

\section{Discussion}

PCOS is a complex condition that affects both the reproductive and the metabolic systems. In this meta-analysis including 12 cross-sectional and case-control studies, for a total of 995 PCOS and 2275 women from Brazil, BMI, waist circumference, blood pressure, glucose, and HOMA-IR were found to be higher in participants with PCOS. Lipid profile was more adverse than that found in non-PCOS women. Even though only observational 


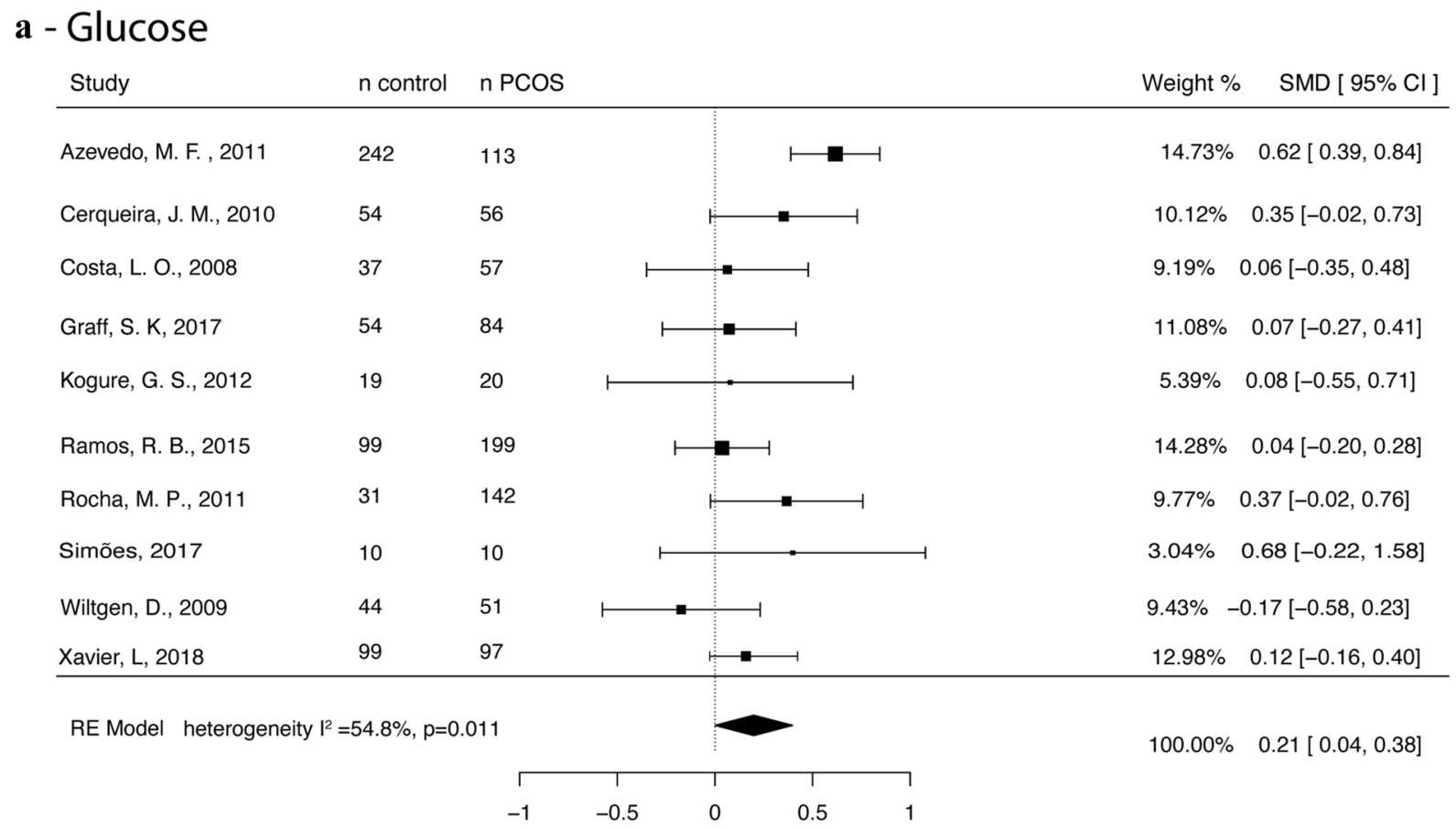

\section{b - HOMA-IR}

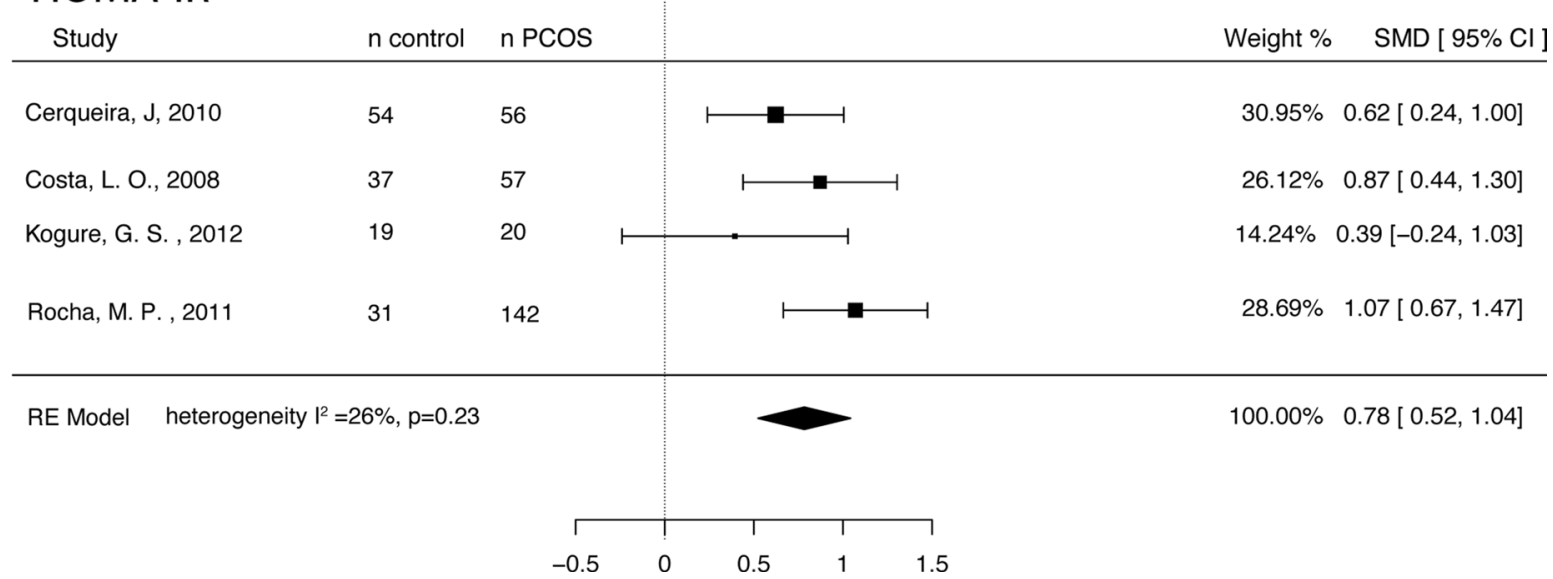

Fig. 3 Forest plot showing a fasting glucose, b HOMA-IR

studies including mostly small samples were examined, the evidence indicates that women with PCOS from different regions of Brazil have a worse cardiometabolic profile than women without PCOS. In addition, the systematic review of 27 observational studies with PCOS women from Brazil indicates that metabolic comorbidities, such as obesity, metabolic syndrome and IFG/IGT are prevalent in Brazilian women with PCOS with no important regional differences. To the best of our knowledge, this is the first systematic review and meta-analysis evaluating metabolic characteristics of women with PCOS in the different regions of Brazil.

Despite the efforts to assess the impact of ethnicity and sociocultural backgrounds on the metabolic traits of PCOS by comparing different populations, very few data are available regarding Latin American countries. In Brazil, the overall ancestry proportion has been described as 0.62 European, 0.21 African and 0.17 Amerindian [51, 52]. However, ancestry proportions seem to differ according to region, as indicated by self-reported 


\begin{tabular}{|c|c|c|c|c|c|}
\hline \multirow{2}{*}{$\begin{array}{l}\text { a- Triglycerides } \\
\qquad \begin{array}{l}\text { Study } \\
\text { Azevedo, M. F., } 2011\end{array}\end{array}$} & \multirow{2}{*}{$\begin{array}{l}n \text { control } \\
242\end{array}$} & \multicolumn{2}{|c|}{$\mathrm{nPCOS}$} & \multicolumn{2}{|c|}{ Weight \% SMD $[95 \% \mathrm{Cl}]$} \\
\hline & & 113 & $\longmapsto$ & $29.74 \%$ & $0.59[0.37,0.82]$ \\
\hline Cerqueira, ১, 2010 & 54 & 56 & $\longmapsto$ & $20.45 \%$ & $0.58[0.20,0.96]$ \\
\hline Costa, L. O., 2008 & 37 & 57 & 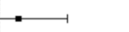 & $18.81 \%$ & $0.16[-0.25,0.58]$ \\
\hline Kogure, G. S., 2012 & 19 & 20 & & $10.91 \%$ & $0.53[-0.11,1.17]$ \\
\hline Rocha, M. P., 2011 & 31 & 142 & $\longrightarrow$ & $20.09 \%$ & $0.03[-0.36,0.42]$ \\
\hline RE Model heterogenei & $3 \%, p=0.079$ & & $\sim$ & $100.00 \%$ & $0.39[0.14,0.64]$ \\
\hline
\end{tabular}

b- HDL- C

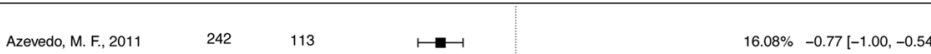

Cerqueira, J, $2010 \quad 54 \quad 56 \quad \square \quad$ 12.22\% $-0.72[-1.10,-0.33]$

Costa, L. $0.2008 \quad 37 \quad 57 \quad \longrightarrow \quad 11.06 \%-0.97[-1.40,-0.53]$

Graff, S. K., $2017 \quad 54 \quad 84 \quad \longmapsto \quad 13.20 \%-0.41[-0.75,-0.06]$

Kogure, G. S., $2012 \quad 19 \quad 20 \quad 7.44 \%-0.47[-1.10,0.17\}$

Radavelli-Bagatini, S., $2013 \quad 1500 \quad 80 \quad \mapsto-16 \quad 19 \%-0.58[-0.80,-0.35]$

Rocha, M. P., $2011 \quad 31 \quad 142 \quad \longrightarrow \quad 12.01 \%-0.65[-1.04,-0.25]$

Wiltgen, D., $2009 \quad 44 \quad 51 \quad 11.79 \% \quad 0.15[-0.26,0.55]$

\begin{tabular}{l|l}
\hline RE Model heterogeneity $\mathrm{l}^{2}=68 \%, \mathrm{p}=0.006$ & $100.00 \%-0.56[-0.78,-0.34]$
\end{tabular}

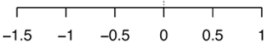

c-LDL-c

Study n control $n$ PCOS

Weight \% SMD $[95 \% \mathrm{Cl}]$

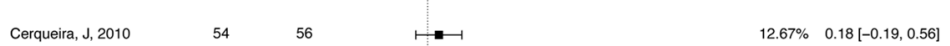

$\begin{array}{llllll}\text { Costa, L. O. } 2008 & 37 & 57 & \longmapsto & 11.99 \% & 0.53[0.11,0.95]\end{array}$

$\begin{array}{lllllll}\text { Gratt, S. K., } 2017 \quad 54 \quad 84 & =13.15 \% & 0.04[-0.30,0.38]\end{array}$

Kogure, G.S., $2012 \quad 19 \quad 20 \quad \square$. $\quad 9.09 \%-0.12[-0.74,0.51]$

\begin{tabular}{lll|lll} 
Radavelli-Bagatini, S. 2013 & 1500 & 80 & $14.67 \%$ & $1.15[0.93,1.38]$
\end{tabular}

$\begin{array}{lllllll}\text { Rocha, M. P., } 2011 & 31 & 142 & - & 12.43 \% & 0.38[-0.01,0.77]\end{array}$

$\begin{array}{lllllll}\text { Witgen, D., } 2009 & 44 & 51 & \bullet & 12.05 \% & 0.72[0.31,1.14]\end{array}$

\begin{tabular}{lllllll} 
Xavier, L., 2018 & 99 & 97 & $13.95 \%$ & 0.50 & {$[0.21,0.78]$} \\
\hline
\end{tabular}

RE Model heterogeneity $\mathrm{l}^{\mathrm{P}}=80.31 \%, \mathrm{p}=<0.0001 \quad 100.00 \% \quad 0.45[0.17,0.74]$

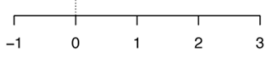

d- Total Cholesterol

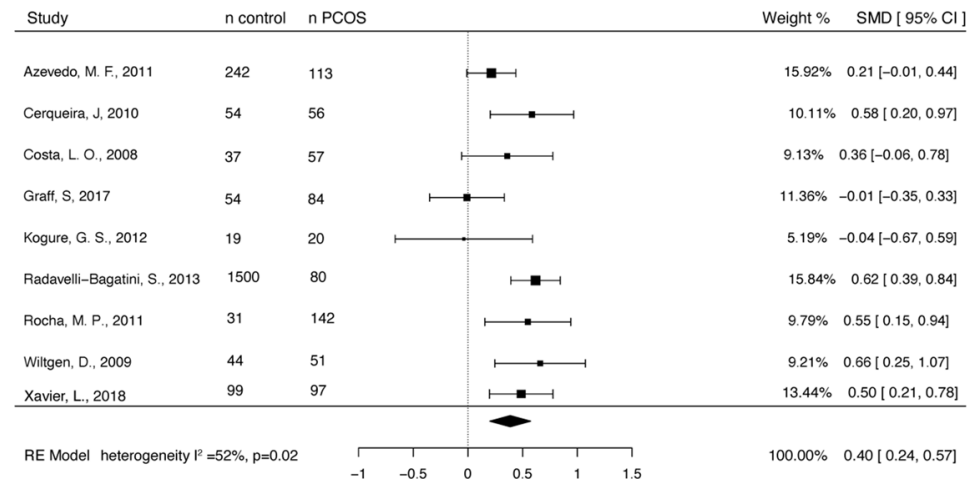

Fig. 4 Forest plot showing a TGL, b HDL-Chol, c LDL-Chol, $\mathbf{d}$ total cholesterol 

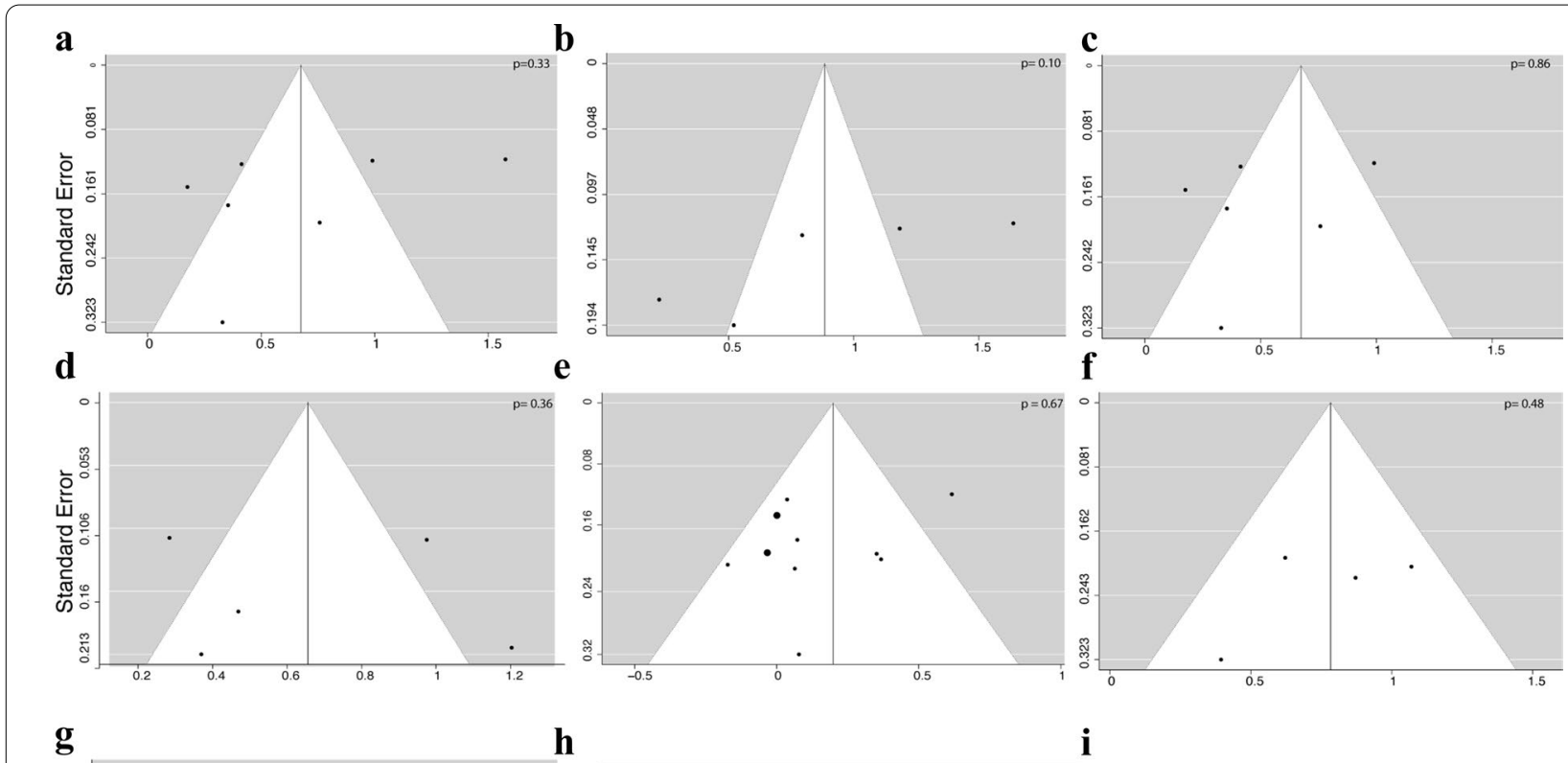

h
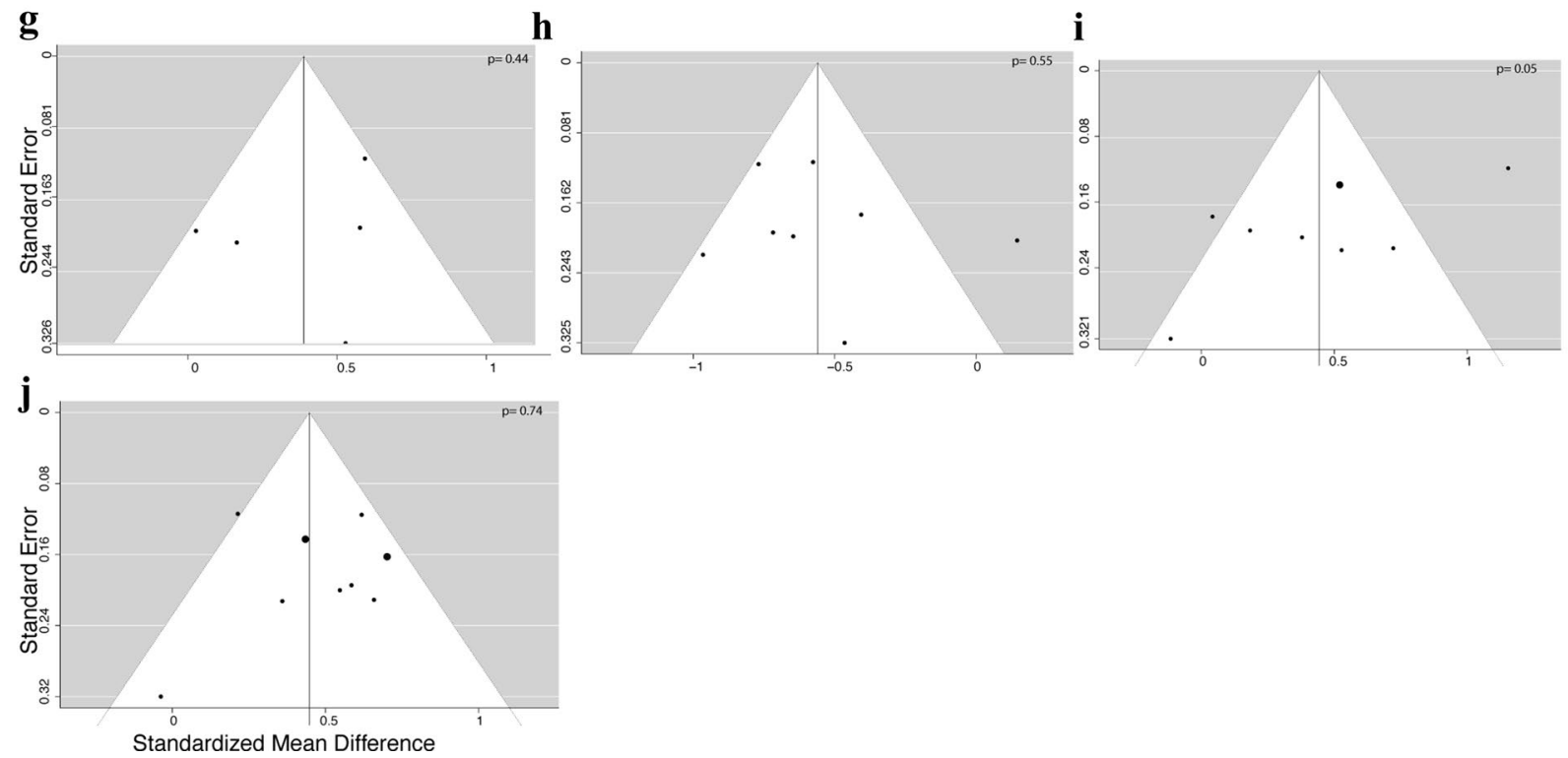

Fig. 5 Funnel plots for risk of publication bias for a BMI, $\mathbf{b}$ waist circumference, $\mathbf{c}$ systolic blood pressure, $\mathbf{d}$ diastolic blood pressure, e glucose, $\mathbf{f}$ HOMA-IR, $\mathbf{g}$ TGL, $\mathbf{h}$ HDL-chol, i LDL-chol, $\mathbf{j}$ total cholesterol

skin color rates, in which lower rates of white are found in the North and Northeastern regions (23 and 29\% respectively) compared to the South, Southeast and Midwest (78, 55 and $48 \%$, respectively) (sidra.ibge.gov.br/ Tabela/3175 - accessed on 06/24/2020). Although these genetic backgrounds could impact the phenotypic heterogeneity of PCOS, the evidence emerging from the present study rather suggests similarities in metabolic traits throughout the different regions of the country, a finding that could potentially inform public health care systems, preventive programs, and policies targeting women with PCOS in Brazil.

In the present systematic review and meta-analysis, women with PCOS from Brazil had higher BMI and worse metabolic status. Analysis of the available data showed that in women with PCOS, obesity, metabolic syndrome, and IFG/IGT were more frequent than in Brazilian women from the general population of same age [50]. Of note, most of these studies were from referral populations, which may have influenced the prevalence of comorbidities at least to a certain extent [53]. 
While the current information on the frequency of obesity in women with PCOS from different countries comes from only a few studies, generally with a small number of participants, or from studies reporting obesity as a secondary outcome, the present data could indicate that the prevalence of obesity in Brazilian women with PCOS, varying from 31.6 to $56.6 \%$, may be close to that of Scandinavian countries (42\% [54]; $35 \%$ [55]), and half way between that observed in U.S. women with PCOS diagnosed with Rotterdam criteria $(65 \%)[11,15]$ and that found in Mediterranean countries $(8$ and $31 \%)[6,7,11]$. The prevalence of metabolic syndrome (24.6-42.9\% in Brazilian women with PCOS) seems to be similar to that found in the U.S. (34.6\% [15]; 43\% [13]), and much higher than that observed in Mediterranean countries (10\% [6]; 6.6\% [7]).

The data regarding type 2 diabetes were too few for analysis, with only one study reporting a prevalence of diabetes of $3.6 \%$ in 195 Brazilian women with PCOS. Thus, further studies are needed in order to determine the frequency of diabetes in women with PCOS across the country [56]. In turn, the prevalence of impaired fasting glucose in PCOS women from the South and Northeast of Brazil was similar to that of Mediterranean countries [7] and lower than that observed in the U.S. [12-14].

Taken together, these data suggest that PCOS-related metabolic alterations are less prevalent in Brazil than in the U.S., where the mean body weight of the general population is higher than in most other countries [57]. Also, additional studies are warranted, covering other underrepresented regions, such as the North of Brazil.

Another metabolic feature of PCOS is insulin resistance, with higher risk of impaired glucose tolerance, type 2 diabetes, and gestational diabetes [58-60]. A recent study showed a pooled PCOS prevalence of $24 \%$ $(95 \%$ CI 15 ; 34) in adolescent and adult women with type 1 diabetes, which is markedly higher than the general population [61]. In our systematic review and meta-analysis, we found Brazilian women with PCOS presented increased HOMA-IR. Previous studies have found that women with PCOS, as opposed to controls, have significantly decreased insulin sensitivity with increasing BMI $[58,62]$ and low-grade chronic inflammation $[63,64]$. Previous meta-analyses including different populations have shown higher odds for MetS among women with PCOS $[65,66]$. However, in both studies very few data from Latin American populations were available. In turn, due to the paucity of available data, additional studies assessing prediabetes and diabetes and distinct PCOS phenotypes in different countries of Latin America are warranted and could produce relevant information for the primary and secondary prevention of these PCOS-related metabolic comorbidities in the region.

Considering the dearth of information, the present systematic review and meta-analysis provides a comprehensive overview of metabolic and anthropometric variables among women diagnosed with PCOS in Brazil. A major strength of our study is the extensive search strategy, covering the main databases to avoid missing any relevant information, with active search for studies published also in Portuguese language. Limitations are the small number of studies in view of the size of the region, the small sample sizes, and the possible sources of heterogeneity across the studies. However, there are no other similar analyses in the literature. Thus, this study represents the first evidence to characterize the metabolic profile of women with PCOS in the context of ethnicities and sociocultural backgrounds in Brazil.

\section{Conclusions}

The present results indicate that women with PCOS from different regions of Brazil have worse anthropometric and metabolic profiles than women from the same regions without PCOS. The prevalence of metabolic changes is intermediate in Brazil in comparison with other countries. Regarding the prevalence of diabetes, the evidence produced is not conclusive, suggesting that additional studies are warranted and could produce invaluable results in the context of PCOS.

\section{Supplementary Information}

The online version contains supplementary material available at https://doi. org/10.1186/s13098-021-00636-5.

Additional file 1. Medical subject headings (MeSH) used in the search.

\section{Abbreviations}

BMI: Body mass index; DBP: Diastolic blood pressure; DM: Diabetes mellitus; HDL: High-density lipoprotein; HOMA: Homeostatic Model Assessment; IFG: Impaired fasting glucose; IGT: Impaired glucose tolerance; IR: Insulin resistance; LDL: Low-density lipoprotein; MetS: Metabolic syndrome; NOS: Newcastle-Ottawa scale; PCOS: Polycystic ovary syndrome; SBP: Systolic blood pressure; SMD: Standardized mean difference; TC: Total cholesterol; TGL:

Triglycerides; WC: Waist circumference.

\section{Acknowledgements}

Not applicable

\section{Authors' contributions}

PMS was involved in the conception and design of the study, data collection and analysis, drafted the article and final review. RBR contributed to study design, was involved with data collection and analysis, performed the statistical analysis and drafted the article. LBM contributed to study design, was involved with data collection and analysis, drafted the article and final review. MO was involved in data collection and analysis. EC revised critically the manuscript for important intellectual content. All authors have approved the submitted manuscript. 


\section{Funding}

This work was funded by the Conselho Nacional de Desenvolvimento Científico e Tecnológico (grant number INCT/CNPq 465482/2014-7) and Fundação de Amparo à Pesquisa do Estado do Rio Grande do Sul (grant number INCT/ FAPERGS: 17/2551-0000519-8). The funding source had no role in the collection, analysis, interpretation of data and in the writing of the report or in the decision to submit the article for publication.

\section{Availability of data and materials}

All data generated or analyzed during this study are included within the article and its supplementary information file.

\section{Ethics approval and consent to participate}

Not applicable.

\section{Consent for publication}

Not applicable.

\section{Competing interests}

The authors declare that they have no competing interests.

\section{Author details}

${ }^{1}$ Gynecological Endocrinology Unit, Division of Endocrinology, Hospital de Clínicas de Porto Alegre, Porto Alegre, RS, Brazil. ${ }^{2}$ Department of Physiology, Universidade Federal Do Rio Grande Do Sul, Porto Alegre, RS, Brazil. 3 Post-Graduate Program in Endocrinology, Medicine School, Universidade Federal Do Rio Grande Do Sul, Porto Alegre, RS, Brazil. ${ }^{4}$ Instituto de Medicina Integral Professor Fernando Figueira Hospital, Recife, Pernambuco, Brazil. ${ }^{5}$ University of Palermo School of Medicine, Palermo, Italy. ${ }^{6}$ Present Address: Department of Molecular and Cellular Physiology, Albany Medical College, Albany, NY, USA. ${ }^{7}$ Division of Endocrinology, Hospital de Clínicas de Porto Alegre, Rua Ramiro Barcelos, 2350, Porto Alegre, RS 90035-003, Brazil.

Received: 14 December 2020 Accepted: 3 February 2021

Published online: 16 February 2021

\section{References}

1. Bozdag G, Mumusoglu S, Zengin D, Karabulut E, Yildiz BO. The prevalence and phenotypic features of polycystic ovary syndrome: a systematic review and meta-analysis. Hum Reprod. 2016;31:2841-55.

2. March WA, Moore VM, Willson KJ, Phillips DI, Norman RJ, Davies MJ. The prevalence of polycystic ovary syndrome in a community sample assessed under contrasting diagnostic criteria. Hum Reprod. 2010:25:544-51.

3. Azziz R, Carmina E, Chen Z, Dunaif A, Laven JS, Legro RS, Lizneva D, Natterson-Horowtiz B, Teede HJ, Yildiz BO. Polycystic ovary syndrome. Nat Rev Dis Primers. 2016;2:16057.

4. Revised 2003 consensus on diagnostic criteria and long-term health risks related to polycystic ovary syndrome. Fertil Steril 2004, 81:19-25.

5. Guastella E, Longo RA, Carmina E. Clinical and endocrine characteristics of the main polycystic ovary syndrome phenotypes. Fertil Steril. 2010;94:2197-201.

6. Yildiz BO, Bozdag G, Yapici Z, Esinler I, Yarali H. Prevalence, phenotype and cardiometabolic risk of polycystic ovary syndrome under different diagnostic criteria. Hum Reprod. 2012;27:3067-73.

7. Carmina E, Nasrallah MP, Guastella E, Lobo RA. Characterization of metabolic changes in the phenotypes of women with polycystic ovary syndrome in a large Mediterranean population from Sicily. Clin Endocrinol (Oxf). 2019:91:553-60

8. Teede HJ, Misso ML, Costello MF, Dokras A, Laven J, Moran L, Piltonen T, Norman RJ. Recommendations from the international evidence-based guideline for the assessment and management of polycystic ovary syndrome. Clin Endocrinol (Oxf) 2018.

9. Shroff R, Syrop CH, Davis W, Van Voorhis BJ, Dokras A. Risk of metabolic complications in the new PCOS phenotypes based on the Rotterdam criteria. Fertil Steril. 2007:88:1389-95.

10. Yilmaz M, Isaoglu U, Delibas IB, Kadanali S. Anthropometric, clinical and laboratory comparison of four phenotypes of polycystic ovary syndrome based on Rotterdam criteria. J Obstet Gynaecol Res. 2011;37:1020-6.
11. Clark NM, Podolski AJ, Brooks ED, Chizen DR, Pierson RA, Lehotay DC, Lujan ME. Prevalence of polycystic ovary syndrome phenotypes using updated criteria for polycystic ovarian morphology: an assessment of over 100 consecutive women self-reporting features of polycystic ovary syndrome. Reprod Sci. 2014;21:1034-43.

12. Ehrmann DA, Barnes RB, Rosenfield RL, Cavaghan MK, Imperial J. Prevalence of impaired glucose tolerance and diabetes in women with polycystic ovary syndrome. Diabetes Care. 1999;22:141-6.

13. Legro RS, Kunselman AR, Dodson WC, Dunaif A. Prevalence and predictors of risk for type 2 diabetes mellitus and impaired glucose tolerance in polycystic ovary syndrome: a prospective, controlled study in 254 affected women. J Clin Endocrinol Metab. 1999:84:165-9.

14. Apridonidze T, Essah PA, luorno MJ, Nestler JE. Prevalence and characteristics of the metabolic syndrome in women with polycystic ovary syndrome. J Clin Endocrinol Metab. 2005;90:1929-35.

15. Roe A, Hillman J, Butts S, Smith M, Rader D, Playford M, Mehta NN, Dokras A. Decreased cholesterol efflux capacity and atherogenic lipid profile in young women with PCOS. J Clin Endocrinol Metab. 2014;99:E841-847.

16. Li R, Zhang Q, Yang D, Li S, Lu S, Wu X, Wei Z, Song X, Wang X, Fu S, et al. Prevalence of polycystic ovary syndrome in women in China: a large community-based study. Hum Reprod. 2013;28:2562-9.

17. Satyaraddi A, Cherian KE, Kapoor N, Kunjummen AT, Kamath MS, Thomas N, Paul TV. Body composition, metabolic characteristics, and insulin resistance in obese and nonobese women with polycystic ovary syndrome. J Hum Reprod Sci. 2019;12:78-84.

18. Mohan V, Sandeep S, Deepa R, Shah B, Varghese C. Epidemiology of type 2 diabetes: Indian scenario. Indian J Med Res. 2007:125:217-30.

19. Essah PA, Nestler JE, Carmina E. Differences in dyslipidemia between American and Italian women with polycystic ovary syndrome. J Endocrinol Invest. 2008;31:35-41.

20. Higgins JP, Thompson SG, Deeks JJ, Altman DG. Measuring inconsistency in meta-analyses. BMJ. 2003;327:557-60.

21. Egger M, Davey Smith G, Schneider M, Minder C. Bias in meta-analysis detected by a simple, graphical test. BMJ. 1997;315:629-34.

22. Viechtbauer W. Conducting Meta-Analyses in $\mathrm{R}$ with the metafor Package. 2010, 36:48.

23. Azevedo MF, Costa EC, Oliveira Al, Silva IB, Marinho JC, Rodrigues JA, Azevedo GD. Elevated blood pressure in women with polycystic ovary syndrome: prevalence and associated risk factors. Rev Bras Ginecol Obstet. 2011:33:31-6.

24. Cerqueira JM, Costa LO, Nogueira Ade A, Silva DC, Torres Dde O, Santos AC. Homocysteinemia in polycystic ovary syndrome women. Rev Bras Ginecol Obstet. 2010;32:126-32

25. Costa LO, dos Santos MP, Oliveira M, Viana A. Low-grade chronic inflammation is not accompanied by structural arterial injury in polycystic ovary syndrome. Diabetes Res Clin Pract. 2008;81:179-83.

26. Gabrielli L, Aquino EM. Polycystic ovary syndrome in Salvador, Brazil: a prevalence study in primary healthcare. Reprod Biol Endocrinol. 2012;10:96

27. Soares NP, Santos AC, Costa EC, Azevedo GD, Damasceno DC, Fayh AP, Lemos TM. Diet-induced weight loss reduces DNA damage and cardiometabolic risk factors in overweight/obese women with polycystic ovary syndrome. Ann Nutr Metab. 2016;68:220-7.

28. Tavares A, Rêgo Barros RC. The prevalence of metabolic syndrome in the different phenotypes of polycystic ovarian syndrome. Rev Bras Ginecol Obstet. 2019;41:37-43.

29. Costa EC, Sa JC, Soares EM, Lemos TM, Maranhao TM, Azevedo GD. Anthropometric indices of central obesity how discriminators of metabolic syndrome in Brazilian women with polycystic ovary syndrome. Gynecol Endocrinol. 2012;28:12-5.

30. Avila MA, Bruno RV, Barbosa FC, Andrade FC, Silva AC, Nardi AE. Polycystic ovary syndrome: implications of metabolic dysfunction. Rev Col Bras Cir. 2014;41:106-10.

31. Carvalho LML, Ferreira CN, de Oliveira DKD, Rodrigues KF, Duarte RCF, Teixeira MFA, Xavier LB, Candido AL, Reis FM, Silva IFO, et al. Haptoglobin levels, but not Hp1-Hp2 polymorphism, are associated with polycystic ovary syndrome. J Assist Reprod Genet. 2017;34:1691-8.

32. Kogure GS, Piccki FK, Vieira CS, Martins Wde P, dos Reis RM. Analysis of muscle strength and body composition of women with polycystic ovary syndrome. Rev Bras Ginecol Obstet. 2012;34:316-22. 
33. Lauria PB, Del Puerto HL, Reis AM, Candido AL, Reis FM. Low plasma atrial natriuretic peptide: a new piece in the puzzle of polycystic ovary syndrome. J Clin Endocrinol Metab. 2013;98:4882-9.

34. Maciel GA, Moreira RP, Bugano DD, Hayashida SA, Marcondes JA, Gomes LG, Mendonca BB, Bachega TA, Baracat EC. Association of glucocorticoid receptor polymorphisms with clinical and metabolic profiles in polycystic ovary syndrome. Clinics (Sao Paulo). 2014;69:179-84.

35. Melo AS, Vieira CS, Romano LG, Ferriani RA, Navarro PA. The frequency of metabolic syndrome is higher among PCOS Brazilian women with menstrual irregularity plus hyperandrogenism. Reprod Sci. 2011;18:1230-6.

36. Oliveira RdSMd. Redorat RG, Ziehe GH, Mansur VA, Conceição FL: Arterial hypertension and metabolic profile in patients with polycystic ovary syndrome. Revista Brasileira de Ginecologia e Obstetrícia. 2013;35:21-6.

37. Pedroso DC, Melo AS, Carolo AL, Vieira CS, Rosa e Silva AC, dos Reis RM. Frequency and risk factors for metabolic syndrome in adolescents and adults women with polycystic ovary syndrome. Rev Bras Ginecol Obstet. 2012;34:357-61.

38. Pontes AG, Rehme MF, Martins AM, Micussi MT, Maranhão TM, Pimenta WeP, Pontes A. Insulin resistance in women with polycystic ovary syndrome: relationship with anthropometric and biochemical variables. Rev Bras Ginecol Obstet. 2012;34:74-9.

39. Rocha MP, Marcondes JA, Barcellos CR, Hayashida SA, Curi DD, da Fonseca AM, Bagnoli VR, Baracat EC. Dyslipidemia in women with polycystic ovary syndrome: incidence, pattern and predictors. Gynecol Endocrinol. 2011;27:814-9.

40. Santana LF, de Sá MF, Ferriani RA, de Moura MD, Foss MC, dos Reis RM Effect of metformin on the clinical and metabolic assessment of women with polycystic ovary syndrome. Gynecol Endocrinol. 2004;19:88-96.

41. Simoes RS, Soares JM Jr, Simoes MJ, Nader HB, Baracat MCP, Maciel GAR, Serafini PC, Azziz R, Baracat EC. Small leucine-rich proteoglycans (SLRPs) in the endometrium of polycystic ovary syndrome women: a pilot study. J Ovarian Res. 2017;10:54.

42. Xavier LB, Sóter MO, Sales MF, Oliveira DK, Reis HJ, Candido AL, Reis FM, Silva IO, Gomes KB, Ferreira CN. Evaluation of PCSK9 levels and its genetic polymorphisms in women with polycystic ovary syndrome. Gene. 2018;644:129-36.

43. Wiltgen D, Spritzer PM. Variation in metabolic and cardiovascular risk in women with different polycystic ovary syndrome phenotypes. Fertil Steril. 2010;94:2493-6.

44. Graff SK, Mario FM, Magalhaes JA, Moraes RS, Spritzer PM. Saturated fat intake is related to heart rate variability in women with polycystic ovary syndrome. Ann Nutr Metab. 2017;71:224-33.

45. Radavelli-Bagatini S, de Oliveira IO, Ramos RB, Santos BR, Wagner MS, Lecke SB, Gigante DP, Horta BL, Spritzer PM. Haplotype TGTG from SNP $45 \mathrm{~T} / \mathrm{G}$ and $276 \mathrm{G} / \mathrm{T}$ of the adiponectin gene contributes to risk of polycystic ovary syndrome. J Endocrinol Invest. 2013;36:497-502.

46. Ramos RB, Spritzer PM. FTO gene variants are not associated with polycystic ovary syndrome in women from Southern Brazil. Gene. 2015;560:25-9.

47. Wiltgen D, Benedetto IG, Mastella LS, Spritzer PM. Lipid accumulation product index: a reliable marker of cardiovascular risk in polycystic ovary syndrome. Hum Reprod. 2009;24:1726-31.

48. de Medeiros SF, Yamamoto MM, Bueno HB, Belizario D, Barbosa JS. Prevalence of elevated glycated hemoglobin concentrations in the polycystic ovary syndrome: anthropometrical and metabolic relationship in amazonian women. J Clin Med Res. 2014;6:278-86.

49. Wanderley MDS, Pereira LCR, Santos CB, Cunha VSD, Neves MVJ. Association between insulin resistance and cardiovascular risk factors in polycystic ovary syndrome patients. Rev Bras Ginecol Obstet. 2018;40:188-95.

50. Brasil. Ministério da Saúde. Secretaria de Vigilância em Saúde. Departamento de Análise em Saúde e Vigilância de Doenças não Transmissíveis. Vigitel Brazil 2018: surveillance of risk and protective factors for chronic diseases by telephone survey: estimates of frequency and sociodemographic distribution of risk and protective factors for chronic diseases in the capitals of the 26Brazilian states and the Federal District in 2018. Brasilia: Ministério da Saúde; 2018. http://bvsms.saude.gov.br/bvs/publi cacoes/vigitel_brasil_2018_vigilancia_fatores_risco.pdf. Acessed in 2020.

51. Moura RR, Coelho AV, Balbino Vde Q, Crovella S, Brandao LA. Metaanalysis of Brazilian genetic admixture and comparison with other Latin America countries. Am J Hum Biol. 2015;27:674-80.
52. Homburger JR, Moreno-Estrada A, Gignoux CR, Nelson D, Sanchez E, Ortiz-Tello P, Pons-Estel BA, Acevedo-Vasquez E, Miranda P, Langefeld CD, et al. Genomic insights into the ancestry and demographic history of South America. PLoS Genet. 2015;11:e1005602.

53. Lizneva D, Kirubakaran R, Mykhalchenko K, Suturina L, Chernukha G, Diamond MP, Azziz R. Phenotypes and body mass in women with polycystic ovary syndrome identified in referral versus unselected populations: systematic review and meta-analysis. Fertil Steril. 2016;106(1510-1520):e1512.

54. Pelanis R, Mellembakken JR, Sundström-Poromaa I, Ravn P, Morin-Papunen L, Tapanainen JS, Piltonen T, Puurunen J, Hirschberg AL, Fedorcsak P, et al. The prevalence of Type 2 diabetes is not increased in normal-weight women with PCOS. Hum Reprod. 2017;32:2279-86.

55. Rubin KH, Glintborg D, Nybo M, Abrahamsen B, Andersen M. Development and Risk Factors of Type 2 Diabetes in a Nationwide Population of Women With Polycystic Ovary Syndrome. J Clin Endocrinol Metab. 2017; 102:3848-57.

56. Spritzer PM, Marchesan LB, Santos BR, Cureau FV, Oppermann K. Reis RMd, Ferriani RA, Weiss R, Meirelles R, Candido AL, Reis FM: Prevalence and characteristics of polycystic ovary syndrome in Brazilian women: protocol for a nation-wide case-control study. BMJ Open. 2019;9:e029191.

57. Hales CM, Carroll MD, Fryar CD, Ogden CL. Prevalence of obesity and severe obesity among adults: United States, 2017-2018. NCHS Data Brief 2020:1-8.

58. Kakoly NS, Earnest A, Teede HJ, Moran LJ, Joham AE. The impact of obesity on the incidence of type 2 diabetes among women with polycystic ovary syndrome. Diabetes Care. 2019;42:560-7.

59. Diamanti-Kandarakis E, Spritzer PM, Sir-Petermann T, Motta AB. Insulin resistance and polycystic ovary syndrome through life. Curr Pharm Des. 2012;18:5569-76.

60. Kazemi Jaliseh H, Ramezani Tehrani F, Behboudi-Gandevani S, Hosseinpanah F, Khalili D, Cheraghi L, Azizi F. Polycystic ovary syndrome is a risk factor for diabetes and prediabetes in middle-aged but not elderly women: a long-term population-based follow-up study. Fertil Steril. 2017;108:1078-84.

61. Escobar-Morreale HF, Roldán-Martín MB. Type 1 diabetes and polycystic ovary syndrome: systematic review and meta-analysis. Diabetes Care. 2016;39:639-48.

62. Ollila MM, West S, Keinanen-Kiukaaniemi S, Jokelainen J, Auvinen J, Puukka K, Ruokonen A, Jarvelin MR, Tapanainen JS, Franks S, et al. Overweight and obese but not normal weight women with PCOS are at increased risk of Type 2 diabetes mellitus-a prospective population-based cohort study. Hum Reprod. 2017;32:968.

63. Spritzer PM, Lecke SB, Satler F, Morsch DM. Adipose tissue dysfunction, adipokines, and low-grade chronic inflammation in polycystic ovary syndrome. Reproduction. 2015;149:R219-227.

64. Kelly CC, Lyall H, Petrie JR, Gould GW, Connell JM, Sattar N. Low grade chronic inflammation in women with polycystic ovarian syndrome. J Clin Endocrinol Metab. 2001;86:2453-5.

65. Lim SS, Kakoly NS, Tan JWJ, Fitzgerald G, Bahri Khomami M, Joham AE, Cooray SD, Misso ML, Norman RJ, Harrison CL, et al. Metabolic syndrome in polycystic ovary syndrome: a systematic review, meta-analysis and meta-regression. Obes Rev. 2019;20:339-52.

66. Behboudi-Gandevani S, Amiri M, Bidhendi Yarandi R, Noroozzadeh M, Farahmand M, Rostami Dovom M, Ramezani Tehrani F. The risk of metabolic syndrome in polycystic ovary syndrome: a systematic review and meta-analysis. Clin Endocrinol (Oxf). 2018;88:169-84.

\section{Publisher's Note}

Springer Nature remains neutral with regard to jurisdictional claims in published maps and institutional affiliations. 